\title{
Double-soft limits of gluons and gravitons
}

\section{Thomas Klose, ${ }^{a}$ Tristan McLoughlin, ${ }^{b}$ Dhritiman Nandan, ${ }^{a, e}$ Jan Plefka ${ }^{a}$ and Gabriele Travaglini ${ }^{a, c, d}$}

${ }^{a}$ Institut für Physik und IRIS Adlershof, Humboldt-Universität zu Berlin, Zum Großen Windkanal 6, 12489 Berlin, Germany

${ }^{b}$ School of Mathematics, Trinity College Dublin, College Green, Dublin 2, Ireland

${ }^{c}$ Centre for Research in String Theory, School of Physics and Astronomy, Queen Mary University of London, Mile End Road, London E1 4NS, U.K.

${ }^{d}$ Dipartimento di Fisica, Università di Roma "Tor Vergata", Via della Ricerca Scientifica, 100133 Roma, Italy

e Institut für Mathematik und IRIS Adlershof, Humboldt-Universität zu Berli, Zum Großen Windkanal 6, 12489 Berlin, Germany

E-mail: thklose@physik.hu-berlin.de, tristan@maths.tcd.ie, dhritiman@physik.hu-berlin.de, plefka@physik.hu-berlin.de, g.travaglini@qmul.ac.uk

ABSTRACT: The double-soft limit of gluon and graviton amplitudes is studied in four dimensions at tree level. In general this limit is ambiguous and we introduce two natural ways of taking it: a consecutive double-soft limit where one particle is taken soft before the other and a simultaneous limit where both particles are taken soft uniformly. All limits yield universal factorisation formulae which we establish by BCFW recursion relations down to the subleading order in the soft momentum expansion. These formulae generalise the recently discussed subleading single-soft theorems. While both types of limits yield identical results at the leading order, differences appear at the subleading order. Finally, we discuss double-scalar emission in $\mathcal{N}=4$ super Yang-Mills theory. These results should be of use in establishing the algebraic structure of potential hidden symmetries in the quantum gravity and Yang-Mills S-matrix.

KEYwords: Scattering Amplitudes, Gauge Symmetry, Space-Time Symmetries, Supersymmetric gauge theory

ArXiv EPrint: 1504.05558v2 


\section{Contents}

1 Introduction and conclusions 1

2 Single and consecutive double-soft limits 5

2.1 Single-soft limits 5

2.2 Consecutive double-soft limits 6

3 Simultaneous double-soft gluon limits $\quad 9$

3.1 Summary of results 9

$\begin{array}{ll}3.2 & \text { Derivation from BCFW recursion relations } \\ \end{array}$

4 Simultaneous double-soft graviton limits $\quad 17$

$\begin{array}{lll}4.1 \text { Summary of results } & 17\end{array}$

$\begin{array}{ll}4.2 & \text { Derivation from the BCFW recursion relation } \\ \end{array}$

5 Double-soft scalars in $\mathcal{N}=4$ super Yang-Mills $\quad 21$

$\begin{array}{ll}\text { A Sub-subleading terms } & 25\end{array}$

B Supersymmetric Yang-Mills soft limits $\quad 26$

\section{Introduction and conclusions}

The infrared behaviour of gluon and graviton amplitudes displays a universal factorisation into a soft and a hard contribution which makes it an interesting topic of study. As was already noticed in the early days of quantum field theory $[1,2]$, the emission of a single soft gluon or graviton yields a singular soft function linearly divergent in the soft momentum. There is also universal behaviour at the subleading order in a soft momentum expansion both for gluons and photons [1, 3, 4] and, as was discovered only recently, for gravitons [5]. The authors of [5] moreover related the subleading soft graviton functions to a conjectured hidden symmetry of the quantum gravity S-matrix $[6,7]$ which has the form of an extended $\mathrm{BMS}_{4}$ algebra [8-10] known from classical gravitational waves. Similar claims that the Yang-Mills S-matrix enjoys a hidden two-dimensional Kac-Moody type symmetry were made recently [11]. In this picture the scattering amplitudes in four-dimensional quantum field theory are related to correlation functions of a two-dimensional quantum theory living on the sphere at null infinity. This fascinating proposal merits further study.

The subleading soft gluon and graviton theorems were proven using modern on-shell techniques for scattering amplitudes. ${ }^{1}$ They hold in general dimensions [14-17] and their

\footnotetext{
${ }^{1}$ See e.g. $[12,13]$ for a textbook treatment.
} 
form is strongly constrained by gauge and Poincaré symmetry [18, 19]. These results are so far restricted to tree-level. The important loop-level validity and deformations of the theorem were studied in [20-24]. An ambitwistor string model was proposed in [25, 26] which yields the graviton and gluon tree-level S-matrix in the form of their CHY representation $[27,28]$. In this language the soft theorems have an intriguing two-dimensional origin in terms of corresponding limits of the vertex operators on the ambitwistor string world-sheet [29].

Technically the soft theorems are conveniently expressed as an expansion in a small soft scaling parameter $\delta$ multiplying the momentum of the soft particle $p^{\mu}=\delta q^{\mu}$ with $q^{2}=0$. Taking the soft limit of a gluon in a colour-ordered $(n+1)$-point amplitude $A_{n+1}$ yields the soft theorem at tree-level

$$
\lim _{\delta \rightarrow 0} \mathcal{A}_{n+1}=\left(\frac{1}{\delta} S_{\mathrm{YM}}^{(0)}(q)+S_{\mathrm{YM}}^{(1)}(q)\right) \mathcal{A}_{n}+\mathcal{O}(\delta),
$$

where $\mathcal{A}_{n}=\delta^{(4)}\left(\sum_{i=1}^{n} p_{n}\right) A_{n}$ denotes the full amplitude including the momentum preserving delta-function. The soft functions $S_{\mathrm{YM}}^{(n)}(q)$ are universal, in fact $S_{\mathrm{YM}}^{(1)}(q)$ has the form of a differential operator in momenta and polarisations acting on the the amplitude $\mathcal{A}_{n}$. For soft gravitons the universality even extends down to the sub-subleading order

$$
\lim _{\delta \rightarrow 0} \mathcal{M}_{n+1}=\left(\frac{1}{\delta} S_{\text {grav }}^{(0)}(q)+S_{\text {grav }}^{(1)}(q)+\delta S_{\text {grav }}^{(2)}(q)\right) \mathcal{M}_{n}+\mathcal{O}\left(\delta^{2}\right) .
$$

Now $S_{\text {grav }}^{(1)}$ is a first-order and $S_{\text {grav }}^{(2)}$ a second-order differential operator in the hard momenta and polarisations (or equivalently in spinor helicity variables). The leading soft function $S_{\text {grav }}^{(0)}$ has been associated $[6,7]$ to the Ward identity of the super-translation, while the subleading soft function $S_{\text {grav }}^{(1)}$ to that of the Virasoro (or super-rotation) generators of the extended $\mathrm{BMS}_{4}$ symmetry algebra. However, this subleading connection is still not entirely established.

The soft behaviour of the S-matrix is in general connected to its symmetries. Hence exploring the soft behaviour is a means to uncover hidden symmetries in quantum field theories. This is particularly transparent in the soft behaviour of Goldstone bosons of a spontaneously broken symmetry. In this situation the soft limit of a single scalar in the theory leads to a vanishing amplitude known as Adler's zero [30]. The emergence of a hidden symmetry algebra from the soft behaviour of amplitudes has been beautifully demonstrated in [31]: taking the double soft limit for two scalars reveals the algebraic structure and yields a non-vanishing result of the form

$$
\begin{aligned}
\lim _{\delta \rightarrow 0} \mathcal{A}_{n+2}\left(\phi^{i}\left(\delta q_{1}\right), \phi^{j}\left(\delta q_{2}\right), 3, \ldots\right. & n+2) \\
& =\sum_{a=3}^{n+2} \frac{p_{a} \cdot\left(q_{1}-q_{2}\right)}{p_{a} \cdot\left(q_{1}+q_{2}\right)} f^{i j K} T_{K} \mathcal{A}_{n}(3, \ldots n+2)+\mathcal{O}(\delta)
\end{aligned}
$$

where $T_{K}$ is the generator of the invariant subgroup with $\left[T^{i}, T^{j}\right]=f^{i j K} T_{K}$ in a suitable representation for acting on amplitudes. Using this method the authors of [31] demonstrated that the double-soft limit of two scalars in $\mathcal{N}=8$ supergravity gives rise to the 
structure constants of the hidden $E_{7(7)}$ symmetry algebra acting non-linearly on the scalars. Methods for extracting double-soft limits of scalars in $4 \leq \mathcal{N}<8$ supergravity as well as $\mathcal{N}=16$ supergravity in three dimensions were presented in [32]. Single soft scalar limits were also studied as a classification tool for effective field theories in [33]. Recently, the double-soft limits of spin $1 / 2$ particles were studied in a series of theories and related universal double-soft behaviour could be established [34]. Of course, for fermions the single-soft limit vanishes by statistics. Double-soft scalar and photon limits were studied very recently for several classes of four-dimensional theories containing scalar particles in [35] using the CHY representation [27, 28]. Interesting universal double-soft theorems were established.

In summary these results indicate that (i) double-soft limits of massless particles exhibit universal behaviour going beyond the single-soft theorems, and (ii) that the doublesoft limits have the potential to exhibit the algebraic structure of underlying hidden symmetries of the S-matrix. These insights and results set the stage for the present analysis where we lift the universal double-soft theorems of massless spin 0 and spin $1 / 2$ particles to the spin 1 and 2 cases. The central difference now lies in the non-vanishing single-soft limits reviewed above. This entails an ambiguity in the way one takes a double-soft limit of two gluons or gravitons with momenta $\delta_{1} q_{1}$ and $\delta_{2} q_{2}$ :

- One can take a consecutive soft limit in which one first takes $\delta_{2}$ to zero and thereafter $\delta_{1}$.

$$
\operatorname{CSL}(1,2) \mathcal{A}_{n}(3, \ldots, n+2)=\lim _{\delta_{1} \rightarrow 0} \lim _{\delta_{2} \rightarrow 0} \mathcal{A}_{n+2}\left(\delta_{1} q_{1}, \delta_{2} q_{2}, 3, \ldots, n+2\right) .
$$

The ambiguity of this limit is then reflected in a non vanishing anti-symmetrised version of this consecutive limit

$$
\operatorname{aCSL}(1,2) \mathcal{A}_{n}(3, \ldots, n+2)=\frac{1}{2}\left[\lim _{\delta_{1} \rightarrow 0}, \lim _{\delta_{2} \rightarrow 0}\right] \mathcal{A}_{n+2}\left(\delta_{1} q_{1}, \delta_{2} q_{2}, 3, \ldots, n+2\right) .
$$

In fact we shall see that for gluons or gravitons of the same helicity the antisymmetrised consecutive limit always vanishes at leading order. For the case of different helicities of the two soft particles, the anti-symmetrised consecutive limit is non-zero. Such an anti-symmetrised consecutive limit for the case of identical helicity photons and gravitons was recently studied in [29].

- Alternatively one can take a simultaneous soft limit in which one sets $\delta_{1}=\delta_{2}=\delta$ and sends both momenta simultaneously to zero

$$
\operatorname{DSL}(1,2) \mathcal{A}_{n}(3, \ldots, n+2)=\lim _{\delta \rightarrow 0} \mathcal{A}_{n+2}\left(\delta q_{1}, \delta q_{2}, 3, \ldots, n+2\right) .
$$

It is this limit which naturally arises in the scalar scenarios where a single soft limit vanishes due to Adler's zero, and thus also the consecutive double-soft limit.

Both double-soft functions have a leading quadratic divergence in the soft limit. In order to obtain a uniform description we set $\delta_{1}=\delta_{2}=\delta$ also for the consecutive limit after 
having taken the limits. It is then natural to define the subleading double-soft functions via the series

$$
\operatorname{CSL}(1,2)=\sum_{i} \delta^{i-2} \operatorname{CSL}^{(i)}(1,2) \quad \text { and } \quad \operatorname{DSL}(1,2)=\sum_{i} \delta^{i-2} \operatorname{DSL}^{(i)}(1,2)
$$

Universality extends down at least to the subleading order.

It is interesting to compare the two soft-functions. As we shall show at leading order in the case of identical helicities of particles 1 and 2 they agree

$$
\operatorname{CSL}^{(0)}\left(1^{h}, 2^{h}\right)=\operatorname{DSL}^{(0)}\left(1^{h}, 2^{h}\right) .
$$

both for gravity and Yang-Mills. At the subleading order still for the same helicities the two continue to agree in the gravity case but differ in the colour-ordered Yang-Mills case

$$
\operatorname{CSL}_{\text {gravity }}^{(1)}\left(1^{h}, 2^{h}\right)=\operatorname{DSL}_{\text {gravity }}^{(1)}\left(1^{h}, 2^{h}\right) \quad \text { but } \quad \operatorname{CSL}_{\mathrm{YM}}^{(1)}\left(1^{h}, 2^{h}\right) \neq \operatorname{DSL}_{\mathrm{YM}}^{(1)}\left(1^{h}, 2^{h}\right) .
$$

If the two soft particles carry opposite helicities the situation is different. While the leading contributions continue to agree for gravity they now disagree at the leading level also for Yang-Mills

$$
\operatorname{CSL}_{\text {gravity }}^{(0)}\left(1^{h}, 2^{\bar{h}}\right)=\operatorname{DSL}_{\text {gravity }}^{(0)}\left(1^{h}, 2^{\bar{h}}\right) \quad \text { but } \quad \operatorname{CSL}_{\mathrm{YM}}^{(0)}\left(1^{h}, 2^{\bar{h}}\right) \neq \operatorname{DSL}_{\mathrm{YM}}^{(0)}\left(1^{h}, 2^{\bar{h}}\right) .
$$

At the subleading order both gravity and Yang-Mills disagree

$$
\operatorname{CSL}_{\text {gravity }}^{(1)}\left(1^{h}, 2^{\bar{h}}\right) \neq \operatorname{DSL}_{\text {gravity }}^{(1)}\left(1^{h}, 2^{\bar{h}}\right) \quad \text { and } \quad \operatorname{CSL}_{\mathrm{YM}}^{(1)}\left(1^{h}, 2^{\bar{h}}\right) \neq \operatorname{DSL}_{\mathrm{YM}}^{(1)}\left(1^{h}, 2^{\bar{h}}\right) .
$$

These results should be of use for establishing the algebraic structure of potential hidden symmetries in the quantum gravity and Yang-Mills S-matrix. This, however, is left for future work.

As a final application of our work, we use supersymmetric recursion relations [31, 36] in $\mathcal{N}=4$ super Yang-Mills to address double-soft limits. This set-up can be used to re-derive the double-soft limits of gluons obtained from the non-supersymmetric recursion relations, but also to study double-soft scalar emission. The interesting observation here is that while a single-soft scalar limit in $\mathcal{N}=4$ super Yang-Mills is finite, and hence non-universal, double-soft scalar emissions gives rise to a divergence, and we compute the corresponding double-soft scalar function.

The paper is organised as follows. In the next section we first review single-soft limits of gluons and gravitons, and we then apply these results to study consecutive double-soft limits of the same particles. Section 3 and 4 contain the main results of this paper, namely the analysis of simultaneous double-soft limits of gluons and gravitons. Finally, we discuss double-soft scalar emission in section 4. Two appendices with technical details of some of our calculations complete the paper.

Note added: after finishing this work, we were made aware in recent email correspondence with Anastasia Volovich and Congkao Wen of a work of Volovich, Wen and Zlotnikov [37] which has some overlap with our paper. 


\section{Single and consecutive double-soft limits}

We start from an amplitude of $n+1$ particles with momenta $p_{1}$ to $p_{n+1}$ and take the momentum of the first particle to be soft by setting $p_{1}=\delta_{1} q_{1}$ and expanding the amplitude in powers of $\delta_{1}$. In terms of spinor variables, we define the soft limit by $\lambda_{p_{1}}=\sqrt{\delta_{1}} \lambda_{q_{1}}$ and $\tilde{\lambda}_{p_{1}}=\sqrt{\delta_{1}} \tilde{\lambda}_{q_{1}}$.

In order to keep the notation compact, we will use $\lambda_{q_{1}} \equiv \lambda_{1} \equiv|1\rangle$ and $\left.\tilde{\lambda}_{q_{1}} \equiv \tilde{\lambda}_{1} \equiv \mid 1\right]$ for the soft particle and $\lambda_{p_{a}} \equiv \lambda_{a} \equiv|a\rangle$ and $\left.\tilde{\lambda}_{p_{a}} \equiv \tilde{\lambda}_{a} \equiv \mid a\right]$ for the hard ones $a=2, \ldots, n+1$.

\subsection{Single-soft limits}

Yang-Mills. The single-soft limit, including the subleading term, for color-ordered YangMills amplitudes is given by $[1,3,4]$

$$
\begin{aligned}
A_{n+1}\left(1^{h_{1}}, 2, \ldots, n+1\right) & \\
& =\left[\frac{1}{\delta_{1}} S^{(0)}\left(n+1,1^{h_{1}}, 2\right)+S^{(1)}\left(n+1,1^{h_{1}}, 2\right)+\ldots\right] A_{n}(2, \ldots, n+1),
\end{aligned}
$$

with

$$
\begin{aligned}
S^{(0)}\left(n+1,1^{+}, 2\right) & =\frac{\langle n+12\rangle}{\langle n+11\rangle\langle 12\rangle}, \\
S^{(1)}\left(n+1,1^{+}, 2\right) & =\frac{1}{\langle 12\rangle} \tilde{\lambda}_{1}^{\dot{\alpha}} \frac{\partial}{\partial \tilde{\lambda}_{2}^{\dot{\alpha}}}+\frac{1}{\langle n+11\rangle} \tilde{\lambda}_{1}^{\dot{\alpha}} \frac{\partial}{\partial \tilde{\lambda}_{n+1}^{\dot{\alpha}}}
\end{aligned}
$$

for a positive-helicity gluon. For a negative-helicity gluon the soft factors are given by conjugation of the spinor variables, $\lambda_{i} \leftrightarrow \tilde{\lambda}_{i}$.

Gravity. For the gravitational case we have $[2,5]$

$$
\begin{aligned}
\mathcal{M}_{n+1}\left(1^{h_{1}}, 2, \ldots,\right. & n+1) \\
& =\left[\frac{1}{\delta_{1}} S^{(0)}\left(1^{h_{1}}\right)+S^{(1)}\left(1^{h_{1}}\right)+\delta_{1} S^{(2)}\left(1^{h_{1}}\right)+\ldots\right] \mathcal{M}_{n}(2, \ldots, n+1),
\end{aligned}
$$

where for a positive-helicity graviton

$$
S^{(0)}\left(1^{+}\right)=\sum_{a=2}^{n+1} \frac{[1 a]}{\langle 1 a\rangle} \frac{\langle x a\rangle}{\langle x 1\rangle} \frac{\langle y a\rangle}{\langle y 1\rangle}, \quad S^{(1)}\left(1^{+}\right)=\frac{1}{2} \sum_{a=2}^{n+1} \frac{[1 a]}{\langle 1 a\rangle}\left(\frac{\langle x a\rangle}{\langle x 1\rangle}+\frac{\langle y a\rangle}{\langle y 1\rangle}\right) \tilde{\lambda}_{1}^{\dot{\alpha}} \frac{\partial}{\partial \tilde{\lambda}_{a}^{\dot{\alpha}}}
$$

The spinors $\lambda_{x}$ and $\lambda_{y}$ are arbitrary reference spinors. The sub-subleading term is given by

$$
S^{(2)}\left(1^{+}\right)=\frac{1}{2} \sum_{a=2}^{n+1} \frac{[1 a]}{\langle 1 a\rangle} \tilde{\lambda}_{1}^{\dot{\alpha}} \tilde{\lambda}_{1}^{\dot{\beta}} \frac{\partial^{2}}{\partial \tilde{\lambda}_{a}^{\dot{\alpha}} \partial \tilde{\lambda}_{a}^{\dot{\beta}}}
$$

As for the gluonic case, the opposite helicity factors are found by conjugation. 


\subsection{Consecutive double-soft limits}

In all double-soft limits, we start from an amplitude of $n+2$ particles and set the momenta of the first and the second particle to $p_{1}=\delta_{1} q_{1}$ and $p_{2}=\delta_{2} q_{2}$ respectively. In terms of spinor variables, we distribute the $\delta$ 's symmetrically as above: $\left\{\sqrt{\delta_{1}} \lambda_{q_{1}}, \sqrt{\delta_{1}} \tilde{\lambda}_{q_{1}}\right\}$ and $\left\{\sqrt{\delta_{2}} \lambda_{q_{2}}, \sqrt{\delta_{2}} \tilde{\lambda}_{q_{2}}\right\}$.

By expanding the amplitude in $\delta_{1}$ and $\delta_{2}$, we obtain various double-soft limits. In the consecutive soft limit - in contradistinction to the simultaneous double-soft limit to be discussed in the next section - we first expand in $\delta_{2}$ while keeping $\delta_{1}$ fixed, and then expand each term of the series in $\delta_{1}$. The result can be calculated straightforwardly from repeated use of the above single-soft limits.

Yang-Mills. As above, we first consider the case of gluons. Let us define the "consecutive soft limit factor" $\operatorname{CSL}\left(n+2,1^{h_{1}}, 2^{h_{2}}, 3\right)$ by

$$
\begin{aligned}
\operatorname{CSL}\left(n+2,1^{h_{1}}, 2^{h_{2}}, 3\right) A_{n}(3, \ldots, n+2) \\
\equiv \lim _{\delta_{1} \rightarrow 0} \lim _{\delta_{2} \rightarrow 0} A_{n+2}\left(\delta_{1} q_{1}^{h_{1}}, \delta_{2} q_{2}^{h_{2}}, 3, \ldots, n+2\right) \\
=\left[\frac{1}{\delta_{2}} S^{(0)}\left(1,2^{h_{2}}, 3\right)+S^{(1)}\left(1,2^{h_{2}}, 3\right)\right] \times \\
\quad \times\left[\frac{1}{\delta_{1}} S^{(0)}\left(n+2,1^{h_{1}}, 3\right)+S^{(1)}\left(n+2,1^{h_{1}}, 3\right)\right] A_{n}(3, \ldots, n+2) .
\end{aligned}
$$

We can also define symmetrised and antisymmetrised versions of the consecutive limits

$$
\begin{aligned}
& \operatorname{sCSL}\left(n+2,1^{h_{1}}, 2^{h_{2}}, 3\right) A_{n}(3, \ldots, n+2) \equiv \frac{1}{2}\left\{\lim _{\delta_{1} \rightarrow 0}, \lim _{\delta_{2} \rightarrow 0}\right\} A_{n+2}\left(\delta_{1} q_{1}^{h_{1}}, \delta_{2} q_{2}^{h_{2}}, 3 \ldots, n+2\right), \\
& \operatorname{aCSL}\left(n+2,1^{h_{1}}, 2^{h_{2}}, 3\right) A_{n}(3, \ldots, n+2) \equiv \frac{1}{2}\left[\lim _{\delta_{1} \rightarrow 0}, \lim _{\delta_{2} \rightarrow 0}\right] A_{n+2}\left(\delta_{1} q_{1}^{h_{1}}, \delta_{2} q_{2}^{h_{2}}, 3 \ldots, n+2\right) .
\end{aligned}
$$

As it will be of interest later, let us consider specific helicities:

$$
\begin{aligned}
& \operatorname{CSL}\left(n+2,1^{+}, 2^{+}, 3\right)=\frac{1}{\delta_{1} \delta_{2}} \frac{\langle n+23\rangle}{\langle n+21\rangle\langle 12\rangle\langle 23\rangle}+\mathcal{O}\left(\delta_{2}^{0} / \delta_{1}, \delta_{1}^{0} / \delta_{2}\right) \\
& \operatorname{CSL}\left(n+2,1^{+}, 2^{-}, 3\right)=\frac{1}{\delta_{1} \delta_{2}} \frac{\langle n+23\rangle}{\langle n+21\rangle[12][23]} \frac{[13]}{\langle 13\rangle}+\mathcal{O}\left(\delta_{2}^{0} / \delta_{1}, \delta_{1}^{0} / \delta_{2}\right)
\end{aligned}
$$

If we take the reverse consecutive limit, i.e. expand first in $\delta_{1}$ and then in $\delta_{2}$, the leading term in $\operatorname{CSL}\left(1^{+}, 2^{+}\right)$is unchanged; hence the symmetric combination is the same as either ordering while the antisymmetric combination vanishes.

It is in fact useful to consider subleading terms; for simplicity, after expanding, we will set $\delta_{1}=\delta_{2}=\delta$ and define

$$
\operatorname{CSL}\left(n+2,1^{h_{1}}, 2^{h_{2}}, 3\right)=\sum_{i} \delta^{i-2} \operatorname{CSL}^{(i)}\left(n+2,1^{h_{1}}, 2^{h_{2}}, 3\right),
$$


and similarly for s/aCSL. The first subleading term is given by

$$
\begin{aligned}
\mathrm{CSL}^{(1)}\left(n+2,1^{+}, 2^{+}, 3\right) \\
\quad=S^{(0)}\left(1,2^{+}, 3\right) S^{(1)}\left(n+2,1^{+}, 3\right)+S^{(1)}\left(1,2^{+}, 3\right) S^{(0)}\left(n+2,1^{+}, 3\right) .
\end{aligned}
$$

As $S^{(1)}$ involves derivatives there will in principle be "contact" terms when they act on the other soft factor, however as the derivatives are only with respect to the $\tilde{\lambda}$ 's and $S^{(0)}$ depends only on the $\lambda$ 's they are trivially zero. ${ }^{2}$

A short calculation yields the symmetric and antisymmetric combination of the consecutive soft factor at the next order

$$
\begin{aligned}
& \mathrm{s} / \mathrm{aCSL}^{(1)}\left(n+2,1^{+}, 2^{+}, 3\right)=+\frac{1}{2}\left(\frac{\langle n+23\rangle\langle 12\rangle \pm\langle n+22\rangle\langle 13\rangle}{\langle 23\rangle\langle n+21\rangle\langle 12\rangle\langle 13\rangle}\right) \tilde{\lambda}_{2}^{\dot{\alpha}} \frac{\partial}{\partial \tilde{\lambda}_{3}^{\dot{\alpha}}} \\
& +\frac{1}{2}\left(\frac{\langle n+22\rangle\langle 13\rangle \pm\langle n+23\rangle\langle 12\rangle}{\langle 23\rangle\langle n+21\rangle\langle 12\rangle\langle n+22\rangle}\right) \tilde{\lambda}_{1}^{\dot{\alpha}} \frac{\partial}{\partial \tilde{\lambda}_{n+2}^{\dot{\alpha}}} \\
& +\frac{1}{2} \frac{\tilde{\lambda}_{1}^{\dot{\alpha}}}{\langle 12\rangle\langle 23\rangle} \frac{\partial}{\partial \tilde{\lambda}_{3}^{\dot{\alpha}}} \pm \frac{1}{2} \frac{\tilde{\lambda}_{2}^{\dot{\alpha}}}{\langle n+21\rangle\langle 12\rangle} \frac{\partial}{\partial \tilde{\lambda}_{n+2}^{\dot{\alpha}}},
\end{aligned}
$$

where the upper sign corresponds to the symmetric case and the lower sign to the antisymmetric case. In the antisymmetric case, the expression can be simplified further,

$$
\begin{aligned}
& \operatorname{aCSL}^{(1)}\left(n+2,1^{+}, 2^{+}, 3\right) \\
&=\frac{1}{2\langle 12\rangle}\left[\left(\frac{\tilde{\lambda}_{1}^{\dot{\alpha}}}{\langle 23\rangle}-\frac{\tilde{\lambda}_{2}^{\dot{\alpha}}}{\langle 13\rangle}\right) \frac{\partial}{\partial \tilde{\lambda}_{3}^{\dot{\alpha}}}-\left(\frac{\tilde{\lambda}_{1}^{\dot{\alpha}}}{\langle 2 n+2\rangle}-\frac{\tilde{\lambda}_{2}^{\dot{\alpha}}}{\langle 1 n+2\rangle}\right) \frac{\partial}{\partial \tilde{\lambda}_{n+2}^{\dot{\alpha}}}\right] .
\end{aligned}
$$

Turning to the case of mixed helicity, the leading term for the reversed limit is already different and so we find

$$
\mathrm{s} / \operatorname{aCSL}^{(0)}\left(n+2,1^{+}, 2^{-}, 3\right)=\frac{1}{2} \frac{1}{\langle n+21\rangle[23]}\left(\frac{\langle n+23\rangle}{[12]} \frac{[13]}{\langle 13\rangle} \pm \frac{[n+23]}{\langle 12\rangle} \frac{\langle 2 n+2\rangle}{[2 n+2]}\right),
$$

where again the upper sign corresponds to the symmetric case, which will be the object most directly comparable to the simultaneous double-soft limit, and the lower sign to the antisymmetric case. At subleading order we find for the symmetric/antisymmetric case

$$
\begin{aligned}
\mathrm{s} \operatorname{aCSL}^{(1)}\left(n+2,1^{+}, 2^{-}, 3\right)= & \pm \frac{1}{2} \frac{1}{[n+22]^{2}} \frac{[n+21]}{\langle n+21\rangle}+\frac{1}{2} \frac{1}{\langle 13\rangle^{2}} \frac{\langle 23\rangle}{[23]} \\
& +\frac{1}{2} \frac{\langle n+23\rangle\langle 12\rangle \pm\langle n+22\rangle\langle 13\rangle}{[23]\langle n+21\rangle\langle 12\rangle\langle 13\rangle} \lambda_{2}^{\alpha} \frac{\partial}{\partial \lambda_{3}^{\alpha}} \\
& +\frac{1}{2} \frac{[n+22][13] \pm[n+23][12]}{[23]\langle n+21\rangle[12][23]} \tilde{\lambda}_{1}^{\dot{\alpha}} \frac{\partial}{\partial \tilde{\lambda}_{n+2}^{\dot{\alpha}}} \\
& +\frac{1}{2} \frac{[13]}{[12][23]} \frac{\tilde{\lambda}_{1}^{\dot{\alpha}}}{\langle 13\rangle} \frac{\partial}{\partial \tilde{\lambda}_{3}^{\dot{\alpha}}} \pm \frac{1}{2} \frac{\langle n+22\rangle}{\langle n+21\rangle\langle 12\rangle} \frac{\lambda_{2}^{\alpha}}{[n+22]} \frac{\partial}{\partial \lambda_{n+2}^{\alpha}} .
\end{aligned}
$$

\footnotetext{
${ }^{2}$ It is perhaps worthwhile to note that this is only valid for generic external momenta as we neglect holomorphic anomaly terms that can arise when external legs are collinear with soft legs.
} 
As before we find some simplifications for the antisymmetric combination of consecutive limits,

$$
\begin{aligned}
\operatorname{aCSL}^{(1)}\left(n+2,1^{+}, 2^{-}, 3\right)= & \frac{1}{2} \frac{1}{\langle 13\rangle^{2}} \frac{\langle 23\rangle}{[23]}-\frac{1}{2} \frac{1}{[n+22]^{2}} \frac{[n+21]}{\langle n+21\rangle} \\
& +\frac{1}{2} \frac{\tilde{\lambda}_{1}^{\dot{\alpha}}}{[12]}\left(\frac{1}{[n+22]} \frac{[n+21]}{\langle n+21\rangle} \frac{\partial}{\partial \tilde{\lambda}_{n+2}^{\dot{\alpha}}}+\frac{1}{[23]} \frac{[13]}{\langle 13\rangle} \frac{\partial}{\partial \tilde{\lambda}_{3}^{\dot{\alpha}}}\right) \\
& -\frac{1}{2} \frac{\lambda_{2}^{\alpha}}{\langle 12\rangle}\left(\frac{1}{\langle n+21\rangle} \frac{\langle n+22\rangle}{[n+22]} \frac{\partial}{\partial \lambda_{n+2}^{\alpha}}+\frac{1}{\langle 13\rangle} \frac{\langle 23\rangle}{[23]} \frac{\partial}{\partial \lambda_{3}^{\alpha}}\right) .
\end{aligned}
$$

Gravity. We can repeat the above considerations for the gravitational case and similarly define the consecutive soft limit factor $\operatorname{CSL}\left(1^{h_{1}}, 2^{h_{2}}\right)$ as first taking particle 2 to be soft and then 1 . If both gravitons have positive helicity we find at leading order

$$
\operatorname{CSL}^{(0)}\left(1^{+}, 2^{+}\right)=S^{(0)}\left(2^{+}\right) S^{(0)}\left(1^{+}\right)=\frac{1}{\langle 12\rangle^{4}} \sum_{a, b \neq 1,2}^{n+2} \frac{[2 a][1 b]}{\langle 2 a\rangle\langle 1 b\rangle}\langle 1 a\rangle^{2}\langle 2 b\rangle^{2},
$$

where we have used the freedom to choose the reference spinors in the two soft factors separately. Specifically, we chose the two reference spinors in $S^{(0)}\left(2^{+}\right)$to be $\lambda_{1}$ and those in $S^{(0)}\left(1^{+}\right)$to be $\lambda_{2}$. This makes the symmetry in particles 1 and 2 manifest, such that

$$
\operatorname{aCSL}^{(0)}\left(1^{+}, 2^{+}\right)=0 \text {. }
$$

We see that the consecutive soft limit naturally involves a double sum over the external legs.

At the next order we have

$$
\mathrm{CSL}^{(1)}\left(1^{+}, 2^{+}\right)=S^{(0)}\left(2^{+}\right) S^{(1)}\left(1^{+}\right)+S^{(1)}\left(2^{+}\right) S^{(0)}\left(1^{+}\right) .
$$

Once again there will in principle be contact terms, which involve only a single sum over external legs, specifically

$$
S^{(1)}\left(2^{+}\right) S^{(0)}\left(1^{+}\right)=\frac{1}{2} \sum_{a \neq 1,2} \frac{[2 a][12]}{\langle 2 a\rangle\langle 12\rangle} \frac{\left\langle x^{\prime} a\right\rangle\left\langle y^{\prime} a\right\rangle}{\left\langle x^{\prime} 1\right\rangle\left\langle y^{\prime} 1\right\rangle}+\text { non-contact terms },
$$

where $x^{\prime}$ and $y^{\prime}$ denote the reference spinors for the first particle. Choosing as above $\lambda_{x^{\prime}}=\lambda_{y^{\prime}}=\lambda_{2}$, we see that this contact term vanishes by momentum conservation. The complete subleading consecutive soft term is thus

$$
\operatorname{CSL}^{(1)}\left(1^{+}, 2^{+}\right)=\frac{1}{\langle 12\rangle^{3}} \sum_{a, b \neq 1,2} \frac{[2 a][1 b]}{\langle 2 a\rangle\langle 1 b\rangle}\langle 1 a\rangle\langle 2 b\rangle\left[\langle 2 b\rangle \tilde{\lambda}_{2}^{\dot{\alpha}} \frac{\partial}{\partial \tilde{\lambda}_{a}^{\dot{\alpha}}}-\langle 1 a\rangle \tilde{\lambda}_{1}^{\dot{\alpha}} \frac{\partial}{\partial \tilde{\lambda}_{b}^{\dot{\alpha}}}\right] .
$$

Due to the absence of the contact term the expression is naturally symmetric in $q_{1}$ and $q_{2}$ and so $\operatorname{aCSL}^{(1)}\left(1^{+}, 2^{+}\right)$also vanishes.

For the case where the first particle has positive helicity but the second has negative we find, for the same choice of reference spinors and to leading order,

$$
\operatorname{CSL}^{(0)}\left(1^{+}, 2^{-}\right)=\frac{1}{\langle 12\rangle^{2}[12]^{2}} \sum_{a, b \neq 1,2}^{n+2} \frac{\langle 2 a\rangle[1 b]}{[2 a]\langle 1 b\rangle}[1 a]^{2}\langle 2 b\rangle^{2} .
$$


A benefit of this choice of reference spinors is that it makes manifest that the order of soft limits does not matter, i.e.

$$
\operatorname{aCSL}^{(0)}\left(1^{+}, 2^{-}\right)=0 .
$$

At subleading order we have, after taking the symmetric combination of soft limits,

$$
\begin{aligned}
\operatorname{sCSL}^{(1)}\left(1^{+}, 2^{-}\right)= & \frac{1}{2\langle 12\rangle[12]} \sum_{a \neq 1,2} \frac{[1 a]^{2}\langle 2 a\rangle^{2}}{\langle 1 a\rangle^{2}[2 a]^{2}}\left\langle a\left|q_{12}\right| a\right] \\
& +\frac{1}{\langle 12\rangle^{2}[12]} \sum_{a, b \neq 1,2} \frac{\langle 2 a\rangle[1 b]}{[2 a]\langle 1 b\rangle}\left[\langle 2 b\rangle^{2}[1 a] \lambda_{2}^{\alpha} \frac{\partial}{\partial \lambda_{a}^{\alpha}}-\langle 1 a\rangle^{2}[2 b] \lambda_{1}^{\alpha} \frac{\partial}{\partial \lambda_{b}^{\alpha}}\right] .
\end{aligned}
$$

We can of course continue to the sub-subleading terms, CSL $^{(2)}$, however as the explicit expressions are involved we relegate them to appendix A. However it is worth nothing that the sub-subleading terms involve a double contact term which has the same scaling as $\mathrm{CSL}^{(1)}$. If we consider the symmetrized version it has the form

$$
\left.\operatorname{sCSL}^{(2)}\right|_{d c}=\frac{1}{2[12]\langle 12\rangle} \sum_{a \neq 1,2}\left(\frac{[1 a]\langle 2 a\rangle^{4}}{\langle 1 a\rangle^{3}}+\frac{\langle 2 a\rangle[1 a]^{4}}{[2 a]^{3}}\right)
$$

which should be combined with with $\left.\operatorname{sCSL}^{(1)}\right|_{c}$ to give

$$
\frac{1}{2\langle 12\rangle[12]} \sum_{a \neq 1,2} \frac{[1 a]^{3}\langle 2 a\rangle^{3}}{\langle 1 a\rangle[2 a]}\left[\frac{1}{\langle a 1\rangle[1 a]}\left(1-\frac{\langle a 2\rangle[2 a]}{\langle a 1\rangle[1 a]}\right)+\frac{1}{\langle a 2\rangle[2 a]}\left(1-\frac{\langle a 1\rangle[1 a]}{\langle a 2\rangle[2 a]}\right)\right] \text {. }
$$

Notably for $\mathrm{CSL}^{(1)}$ the contact term does not vanish and so we have a non-trivial antisymmetric combination

$$
\operatorname{aCSL}^{(1)}\left(1^{+}, 2^{-}\right)=\frac{1}{2\langle 12\rangle[12]} \sum_{a \neq 1,2} \frac{[1 a]^{2}\langle 2 a\rangle^{2}}{\langle 1 a\rangle^{2}[2 a]^{2}}\left\langle a\left|q_{1 \overline{2}}\right| a\right],
$$

where $q_{1 \overline{2}}=q_{1}-q_{2}$. This term is more local than might be naively expected, rather in having the form of a single sum over hard legs it is more like a single-soft factor.

\section{Simultaneous double-soft gluon limits}

\subsection{Summary of results}

In this section we turn to the simultaneous double-soft limits, where we set $\delta_{1}=\delta_{2}=: \delta$ and expand the amplitude in powers of $\delta$. Correspondingly, we define the "double-soft limit factor" by

$$
\operatorname{DSL}\left(n+2,1^{h_{1}}, 2^{h_{2}}, 3\right) A_{n}(3, \ldots, n+2)=\lim _{\delta \rightarrow 0} A_{n+2}\left(\delta q_{1}^{h_{1}}, \delta q_{2}^{h_{2}}, 3, \ldots, n+2\right),
$$

where the corresponding expansion of the double-soft function in $\delta$ is,

$$
\operatorname{DSL}\left(n+2,1^{h_{1}}, 2^{h_{2}}, 3\right)=\sum_{i} \delta^{i-2} \operatorname{DSL}^{(i)}\left(n+2,1^{h_{1}}, 2^{h_{2}}, 3\right) .
$$


The leading double-soft factor for the $1^{+} 2^{+}$helicity configuration may be straightforwardly derived from the formula of the generic MHV gluon amplitude. For the $1^{+} 2^{-}$helicity case, it is sufficient to consider the split-helicity six-point amplitude $A_{6}\left(5^{+}, 6^{+}, 1^{+}, 2^{-}, 3^{-}, 4^{-}\right) .^{3}$ The results are

$$
\begin{aligned}
& \operatorname{DSL}^{(0)}\left(n+2,1^{+}, 2^{+}, 3\right) \\
& \quad=\frac{\langle n+23\rangle}{\langle n+21\rangle\langle 12\rangle\langle 23\rangle}=S^{(0)}\left(n+2,1^{+}, 2\right) S^{(0)}\left(n+2,2^{+}, 3\right), \\
& \operatorname{DSL}^{(0)}\left(n+2,1^{+}, 2^{-}, 3\right) \\
& \quad=\frac{1}{\left\langle n+2\left|q_{12}\right| 3\right]}\left[\frac{1}{2 k_{n+2} \cdot q_{12}} \frac{[n+23]\langle n+22\rangle^{3}}{\langle 12\rangle\langle n+21\rangle}-\frac{1}{2 k_{3} \cdot q_{12}} \frac{\langle n+23\rangle[31]^{3}}{[12][23]}\right],
\end{aligned}
$$

where

$$
q_{12}:=q_{1}+q_{2} .
$$

These formulae were tested numerically using S@M [38] and GGT [39, 40] for a wide range of MHV, NMHV and NNMHV amplitudes from lengths 6 through 14. Importantly these formulae do not have a "local" expression, i.e. they may not be written as a sum over a density depending on the two soft and one hard leg. Both hard legs are entangled. In the next section we will present a derivation of (3.3) and (3.4) based on BCFW recursion relations $[41,42]$.

The sub-leading corrections to (3.3) and (3.4) are also computed via BCFW recursions in the following section and we present the results below:

$$
\begin{aligned}
& \operatorname{DSL}^{(1)}\left(n+2,1^{+}, 2^{+}, 3\right) \\
& =S^{(0)}\left(n+2,1^{+}, 2\right) S^{(1)}\left(n+2,2^{+}, 3\right)+S^{(0)}\left(1,2^{+}, 3\right) S^{(1)}\left(n+2,1^{+}, 3\right), \\
& \operatorname{DSL}^{(1)}\left(n+2,1^{+}, 2^{-}, 3\right) \\
& =S^{(0)}\left(n+2,1^{+}, 2\right) S^{(1)}\left(n+2,2^{-}, 3\right)+S^{(0)}\left(3,2^{-}, 1\right) S^{(1)}\left(n+2,1^{+}, 3\right) \\
& +\frac{\langle 23\rangle[13]}{[32]\langle 12\rangle} \frac{1}{2 p_{3} \cdot q_{12}} \lambda_{2}^{\alpha} \frac{\partial}{\partial \lambda_{3}^{\alpha}}+\frac{\langle n+22\rangle[2 n+2]}{[n+21]\langle 12\rangle} \frac{1}{2 p_{n+2} \cdot q_{12}} \lambda_{2}^{\alpha} \frac{\partial}{\partial \lambda_{n+2}^{\alpha}} \\
& \quad+\frac{[n+21]\langle 2 n+2\rangle}{\langle 1 n+2\rangle[21]} \frac{1}{2 p_{n+2} \cdot q_{12}} \tilde{\lambda}_{1}^{\dot{\alpha}} \frac{\partial}{\partial \tilde{\lambda}_{n+2}^{\dot{\alpha}}}+\frac{[31]\langle 32\rangle}{\langle 13\rangle[21]} \frac{1}{2 p_{3} \cdot q_{12}} \tilde{\lambda}_{1}^{\dot{\alpha}} \frac{\partial}{\partial \tilde{\lambda}_{3}^{\dot{\alpha}}} \\
& \quad+\left.\mathrm{DSL}^{(1)}\left(n+2,1^{+}, 2^{-}, 3\right)\right|_{c},
\end{aligned}
$$

where,

$$
\left.\operatorname{DSL}^{(1)}\left(n+2,1^{+}, 2^{-}, 3\right)\right|_{c}=\frac{\langle n+22\rangle^{2}[1 n+2]}{\langle n+21\rangle} \frac{1}{\left(2 p_{n+2} \cdot q_{12}\right)^{2}}+\frac{[31]^{2}\langle 23\rangle}{[32]} \frac{1}{\left(2 p_{3} \cdot q_{12}\right)^{2}} .
$$

It is interesting to note that the results for both the leading and the sub-leading simultaneous double-soft function for the $1^{+} 2^{+}$gluons are same as the consecutive soft

\footnotetext{
${ }^{3}$ The explicit expression for the latter amplitude can be found e.g. in Exercise 2.2 of [13].
} 


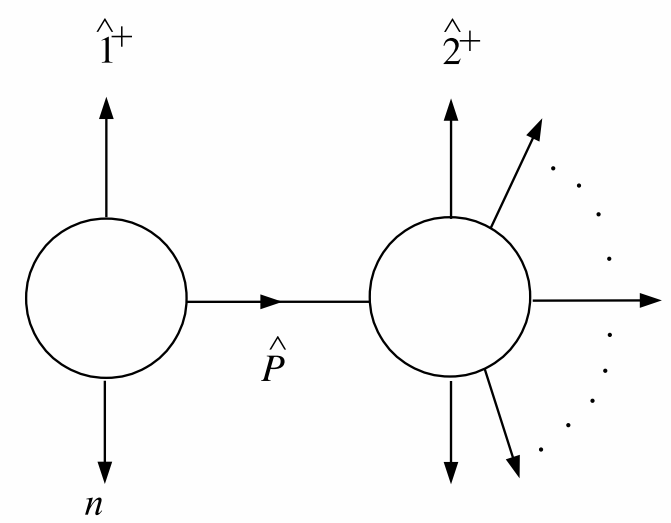

Figure 1. The first BCFW diagram contributing to the double-soft factor. The amplitude on the left-hand side is $\overline{\mathrm{MHV}}$.

limits in the previous section. However, the case with the $1^{+} 2^{-}$is considerably different than the consecutive soft limits scenario and we get new terms especially the last two lines in (3.7) look like some deformation of $S^{(1)}\left(n+2,2^{-}, 3\right)$ and $S^{(1)}\left(n+2,1^{+}, 3\right)$ respectively, due to the double-soft limit. Moreover, we also have the contact terms (3.8) which are absent for the previous case.

\subsection{Derivation from BCFW recursion relations}

In the application of the $\mathrm{BCFW}$ recursion relation we consider a $\langle 12]$ shift, i.e. a holomorphic shift of momentum of the first soft particle and an anti-holomorphic shift of the momentum of the second one, specifically we define

$$
\hat{\lambda}_{1}:=\lambda_{1}+z \lambda_{2}, \quad \hat{\tilde{\lambda}}_{2}:=\tilde{\lambda}_{2}-z \tilde{\lambda}_{1} .
$$

The first observation to make is that generic BCFW diagrams with the soft legs belonging to the left or right $A_{n>3}$ amplitudes are subleading in the soft limit. ${ }^{4}$ This is because the shifted momentum of a soft leg turns hard through the shift in a generic BCFW decomposition. The exception is when any of the two soft legs belongs to a three-point amplitude. Thus nicely, there are two special diagrams to consider, namely those where either one of the two soft particles belongs to a three-point amplitude. In the following we consider separately two cases: $1^{+} 2^{+}$and $1^{+} 2^{-}$.

The $\mathbf{1}^{+} \mathbf{2}^{+}$case. There are two special BCFW diagrams to consider. The first one is shown in figure 1 , where the three-point amplitude sits on the left with the external legs $\hat{1}$ and $n+2$ (with the remaining legs $2, \ldots, n+1$ on the right-hand side). A second diagram has the three-point amplitude on the right-hand side, with external legs $\hat{2}$ and 3 . In the first diagram, the three-point amplitude has the $\overline{\mathrm{MHV}}$ helicity configuration because of our choice of $\langle 12]$ shifts. One easily finds that the solution to $\langle\hat{1} 2\rangle=0$ is

$$
z_{*}=-\frac{\langle 1 n+2\rangle}{\langle 2 n+2\rangle}
$$

\footnotetext{
${ }^{4}$ This observation was made in [31] in relation to the study of a double-soft scalar limit. There, the relevant diagrams turned out to be those involving a four-point functions, and are indeed finite.
} 
and note that $z_{*}$ stays constant as particles 1 and 2 become soft. One also finds

$$
\hat{\lambda}_{1}=-\frac{\langle 12\rangle}{\langle 2 n+2\rangle} \lambda_{n+2},
$$

as well as

$$
\lambda_{\hat{P}} \tilde{\lambda}_{\hat{P}}=\lambda_{n+2}\left(\tilde{\lambda}_{n+2}+\frac{\langle 12\rangle}{\langle n+22\rangle} \tilde{\lambda}_{1}\right)
$$

If we were taking just particle 2 soft, the shifted momentum $\hat{2}$ would remain hard. However we are taking a simultaneous double-soft limit where both particles 1 and 2 are becoming soft, and as a consequence the momentum $\hat{2}$ becomes soft as well, see (3.9) and (3.10). Thus, we can take a soft limit also on the amplitude on the right-hand side. The diagram in consideration then becomes

$$
A_{3}\left((n+2)^{+}, \hat{1}^{+}, \hat{P}^{-}\right) \frac{1}{\left(q_{1}+p_{n+2}\right)^{2}} A_{n}\left(\hat{2}^{+}, \ldots, \hat{P}\right),
$$

Using the explicit expression for the three-point anti-MHV amplitude and the shifts derived earlier, and also (3.12), we may rewrite the right-hand subamplitude in the above with the soft shifted leg $\hat{2}$ as

$$
\begin{aligned}
& A_{n}\left(\hat{2}^{+}, \ldots, p_{n+2}+\right. \delta \frac{\langle 12\rangle}{\langle n+22\rangle}|n+2\rangle[1 \mid) \\
&=e^{\delta \frac{\langle 12\rangle}{\langle n+22\rangle}\left[1 \partial_{n+2}\right]}\left(\frac{1}{\delta} S^{(0)}\left(n+2, \hat{2}^{+}, 3\right)+S^{(1)}\left(n+2, \hat{2}^{+}, 3\right)\right. \\
&\left.+\delta S^{(2)}\left(n+2, \hat{2}^{+}, 3\right)\right) A_{n}(3, \ldots),
\end{aligned}
$$

where, we define,

$$
\left[i \partial_{j}\right]:=\tilde{\lambda}_{i}^{\dot{\alpha}} \frac{\partial}{\partial \tilde{\lambda}_{j}^{\dot{\alpha}}}
$$

From this expressions all relevant leading and subleading contributions to the simultaneous double-soft factor

$$
\begin{aligned}
\operatorname{DSL}\left(n+2,1^{+}, 2^{+}, 3\right)= & \frac{A_{3}\left((n+2)^{+}, \hat{1}^{+}, \hat{P}^{-}\right)}{\left(q_{1}+p_{n+2}\right)^{2}} \\
& \times e^{\delta \frac{\langle 12\rangle}{\langle n+22\rangle}\left[1 \partial_{n+2}\right]}\left(\frac{1}{\delta} S^{(0)}\left(n+2, \hat{2}^{+}, 3\right)+S^{(1)}\left(n+2, \hat{2}^{+}, 3\right)\right. \\
& \left.\quad+\delta S^{(2)}\left(n+2, \hat{2}^{+}, 3\right)\right)
\end{aligned}
$$

may be extracted. Expanding the above expression in $\delta$, at leading order we get,

$$
\operatorname{DSL}^{(0)}\left(n+2,1^{+}, 2^{+}, 3\right)=\frac{\langle n+23\rangle}{\langle n+21\rangle\langle 12\rangle\langle 23\rangle} .
$$

For the sake of definiteness we have considered particle $n+2$ to have positive helicity; a similar analysis can be performed for the case where $n+2$ has negative helicity, and leads to 


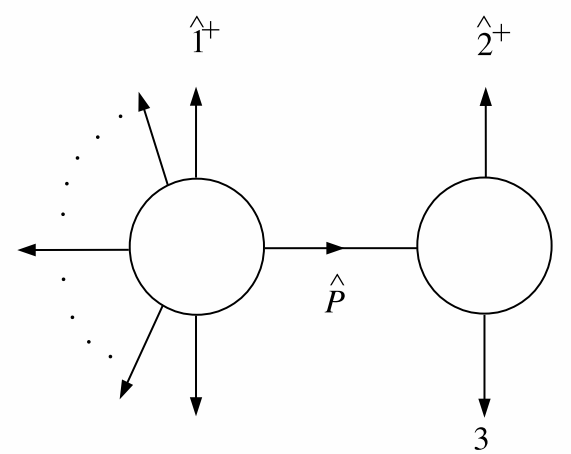

Figure 2. The second BCFW diagram contributing to the double-soft factor. The three-point amplitude is MHV. For the case where gluon 2 has positive helicity we find that this diagram is subleading compared to that in figure 1 and can be discarded; while when 2 has negative helicity this diagram is as leading as figure 1.

the very same conclusions. Note that this contribution (3.13) diverges as $1 / \delta^{2}$ if we scale the soft momenta as $q_{i} \rightarrow \delta q_{i}$, with $i=1,2$. There still is another diagram to compute, shown in figure 2 but we now show that it is in fact subleading. In this diagram, the amplitude on the right-hand side is a three-point amplitude with particles $\hat{2}^{+}, 3$ and $\hat{P}$. If particle 3 has positive helicity, then the three-point amplitude is $\overline{\mathrm{MHV}}$ and hence vanishes because of our shifts. Thus we have to consider only the case when particle 3 has negative helicity. In this case we have the diagram is

$$
A_{3}\left(\hat{2}^{+}, 3^{-}, \hat{P}^{-}\right) \frac{1}{\left(q_{2}+p_{3}\right)^{2}} A_{n+1}\left(\hat{1}^{+}, \hat{P}^{+}, 4, \ldots,(n+2)^{+}\right) .
$$

Similarly to the case discussed earlier, the crucial point is that leg $\hat{1}^{+}$is becoming soft as the momenta 1 and 2 go soft. The diagram then becomes

$$
A_{3}\left(\hat{2}^{+}, 3^{-}, \hat{P}^{-}\right) \frac{1}{\left(q_{2}+p_{3}\right)^{2}} S^{(0)}\left(n+2, \hat{1}^{+}, \hat{P}\right) A_{n}\left(\hat{P}^{+}, 4, \ldots,(n+2)^{+}\right),
$$

and note that $A_{n}\left(\hat{P}^{+}, 4, \ldots,(n+2)^{+}\right) \rightarrow A_{n}\left(3+, 4, \ldots,(n+2)^{+}\right)$in the soft limit. We can now evaluate the prefactor in (3.19) using that, for this diagram, $z_{*}=[23] /[13]$ and

$$
\tilde{\lambda}_{2}=\tilde{\lambda}_{3} \frac{[12]}{[13]}, \quad \lambda_{\hat{P}} \tilde{\lambda}_{\hat{P}}=\left(\lambda_{3}+\frac{[12]}{[13]} \lambda_{2}\right) \tilde{\lambda}_{3} .
$$

In the soft limit we find

$$
A_{3}\left(\hat{2}^{+}, 3^{-}, \hat{P}^{-}\right) \frac{1}{\left(q_{2}+p_{3}\right)^{2}} S^{(0)}\left(n+2, \hat{1}^{+}, \hat{P}\right) \rightarrow \frac{[12]^{3}}{[23][31]} \frac{1}{p_{3} \cdot q_{12}} \frac{\langle n+23\rangle}{\left\langle n+2\left|q_{12}\right| 3\right]},
$$

which is finite under the scaling $q_{i} \rightarrow \delta q_{i}$, with $i=1,2$, and hence subleading with respect to (3.13). In conclusion, we find for the double-soft factor for soft gluons $1^{+} 2^{+}$:

$$
A_{n+2}\left(1^{+}, 2^{+}, 3, \ldots, n\right) \rightarrow \operatorname{DSL}\left(n+2,1^{+}, 2^{+}, 3\right) A_{n}(3, \ldots, n+2),
$$


with

$$
\operatorname{DSL}^{(0)}\left(n+2,1^{+}, 2^{+}, 3\right)=\frac{\langle n+23\rangle}{\langle n+21\rangle\langle 12\rangle\langle 23\rangle},
$$

which agrees with (3.3).

A comment is in order here. We observe that the BCFW diagram in figure 1 is precisely the diagram contributing to the single-soft gluon limit identified originally in [5] and later studied in [4] for Yang-Mills. In the simultaneous double-soft limit, particle $\hat{2}$ also becomes soft thanks to the shifts, and hence we can approximate the BCFW diagram by further extracting a single-soft function for a gluon with soft, shifted momentum $\hat{2}$ :

$$
A_{n+2}\left(1^{+}, 2^{+}, 3, \ldots, n+2\right) \rightarrow S^{(0)}\left(n+2,1^{+}, 2\right) S^{(0)}\left(n+2, \hat{2}^{+}, 3\right) A_{n}(3, \ldots, n+2) .
$$

Moreover, because of our $\langle 12]$ shifts and the holomorphicity of the soft factor for a single positive-helicity gluon, we have that $S^{(0)}\left(n+2, \hat{2}^{+}, 3\right)=S^{(0)}\left(n+2,2^{+}, 3\right)$, thus

$$
\operatorname{DSL}^{(0)}\left(n+2,1^{+}, 2^{+}, 3\right)=S^{(0)}\left(n+2,1^{+}, 2\right) S^{(0)}\left(n+2,2^{+}, 3\right) .
$$

In fact, we can immediately see that a consecutive limit, where particles 1 and 2 are taken soft one after the other (as opposed to our simultaneous double-soft limit) would give the same result. Indeed one would get

$$
\begin{aligned}
A_{n+2}\left(1^{+}, 2^{+}, 3, \ldots, n+2\right) & \rightarrow S^{(0)}\left(n+2,1^{+}, 2\right) A_{n+1}(2, \ldots, n+2) \\
& \rightarrow S^{(0)}\left(n+2,1^{+}, 2\right) S^{(0)}\left(n+2,2^{+}, 3\right) A_{n}(3, \ldots, n+2),
\end{aligned}
$$

in other words at the leading order, the simultaneous double-soft factor for same-helicity soft gluons is nothing but the consecutive soft limit given by the product of two single soft gluon factors.

Now, we present the subleading term in the expansion of (3.16), which scales as $\delta^{-1}$,

$$
\begin{aligned}
\operatorname{DSL}^{(1)}\left(n+2,1^{+}, 2^{+}, 3\right)= & -\frac{\langle n+22\rangle}{\langle n+21\rangle\langle 12\rangle}\left(\frac{1}{\langle 23\rangle} \tilde{\lambda}_{2}^{\dot{\alpha}} \frac{\partial}{\partial \tilde{\lambda}_{3}^{\dot{\alpha}}}+\frac{1}{\langle n+22\rangle} \tilde{\lambda}_{2}^{\dot{\alpha}} \frac{\partial}{\partial \tilde{\lambda}_{n+2}^{\dot{\alpha}}}\right) \\
& -\frac{\langle 13\rangle}{\langle 12\rangle\langle 23\rangle}\left(\frac{1}{\langle 13\rangle} \tilde{\lambda}_{1}^{\dot{\alpha}} \frac{\partial}{\partial \tilde{\lambda}_{3}^{\dot{\alpha}}}+\frac{1}{\langle n+21\rangle} \tilde{\lambda}_{1}^{\dot{\alpha}} \frac{\partial}{\partial \tilde{\lambda}_{n+2}^{\dot{\alpha}}}\right)
\end{aligned}
$$

and the previous equation can be further simplified in terms of leading and subleading terms of single-soft functions as,

$$
\begin{aligned}
\operatorname{DSL}^{(1)}(n+ & \left.2,1^{+}, 2^{+}, 3\right) \\
& =S^{(0)}\left(n+2,1^{+}, 2\right) S^{(1)}\left(n+2,2^{+}, 3\right)+S^{(0)}\left(1,2^{+}, 3\right) S^{(1)}\left(n+2,1^{+}, 3\right) .
\end{aligned}
$$

Note that this contribution was only from the first type of BCFW diagram discussed above, the second type was finite already at the leading order so it again does not contribute to the subleading term here. 
The $\mathbf{1}^{+} \mathbf{2}^{-}$case. We turn again to the two diagrams considered in the previous case. However, we will see that this time they are both leading. Consider the first diagram. The only difference compared to (3.13) is the soft factor, which now has to be replaced with $S^{(0)}\left(\hat{P}, \hat{2}^{-}, 3\right)$ since particle 2 has now negative helicity. We use the same shifts, and make use of the results

$$
\hat{\tilde{\lambda}}_{2}=\frac{q_{12}|n+2\rangle}{\langle 2 n+2\rangle}, \quad \tilde{\lambda}_{\hat{P}}=\frac{\left(q_{1}+p_{n+2}\right)|2\rangle}{\langle 2 n+2\rangle} .
$$

Using this, we evaluate the soft factor as

$$
\frac{[\hat{P} 3]}{[\hat{P} \hat{2}][\hat{2} 3]} \rightarrow \frac{[3|n+2| 2\rangle}{\left[3\left|q_{12}\right| n+2\right\rangle} \frac{\langle n+22\rangle}{2 p_{n+2} \cdot q_{12}} .
$$

The diagram in consideration is then quickly seen to give

$$
\frac{[3 n+2]\langle n+22\rangle^{3}}{\langle 12\rangle\langle n+21\rangle} \frac{1}{\left[3\left|q_{12}\right| n+2\right\rangle} \frac{1}{2 p_{n+2} \cdot q_{12}} A_{n}(3, \ldots, n+2) .
$$

Next we move to the second diagram. Again, in principle one has to distinguish two cases depending on the helicity of particle 3 , but it is easy seen that such cases turn out to give the same result. For the sake of definiteness we illustrate the situation where particle 3 has positive helicity. We obtain

$$
\frac{\langle\hat{P} 2\rangle^{3}}{\langle 23\rangle\langle 3 \hat{P}\rangle} \frac{1}{\langle 23\rangle[32]} S^{(0)}\left(n+2, \hat{1}^{+}, \hat{P}\right) A_{n}(\hat{P}, 4, \ldots, n+2) .
$$

Using

$$
\tilde{\lambda}_{\hat{P}}=\frac{\left[1 \mid\left(q_{2}+p_{3}\right)\right.}{[13]}, \quad \hat{\lambda}_{1}=\frac{\left.q_{12} \mid 3\right]}{[13]},
$$

we easily see that this contribution gives, to leading order in the soft momenta,

$$
\frac{\langle n+23\rangle[13]^{3}}{[12][23]} \frac{1}{\left\langle n+2\left|q_{12}\right| 3\right]} \frac{1}{2 p_{3} \cdot q_{12}} A_{n}(3,4, \ldots, n+2) .
$$

Putting together (3.31) and (3.34) one obtains for the double-soft factor for soft gluons $1^{+} 2^{-}$:

$$
A_{n+2}\left(1^{+}, 2^{-}, 3, \ldots, n\right) \rightarrow \operatorname{DSL}\left(n+2,1^{+}, 2^{-}, 3\right) A_{n}(3, \ldots, n+2),
$$

with

$$
\begin{aligned}
\operatorname{DSL}^{(0)}\left(n+2,1^{+}, 2^{-}, 3\right)=\frac{1}{\left\langle n+2\left|q_{12}\right| 3\right]}\left[\frac{1}{2 p_{n+2} \cdot q_{12}} \frac{[n+23]\langle n+22\rangle^{3}}{\langle 12\rangle\langle n+21\rangle}\right. \\
\left.-\frac{1}{2 p_{3} \cdot q_{12}} \frac{\langle n+23\rangle[31]^{3}}{[12][23]}\right],
\end{aligned}
$$

which agrees with (3.4).

As already observed earlier, we comment that the diagrams in figure 1 and 2 are precisely the BCFW diagrams which would contribute to the single-soft gluon limit when 
either gluon 1 or 2 are taken soft, respectively. Thus, the result we find for the double-soft limit has the structure

$$
\mathrm{DSL}^{(0)}\left(n+2,1^{+}, 2^{-}, 3\right)=S^{(0)}\left(1^{+}\right) S^{(0)}\left(\hat{2}^{-}\right)+S^{(0)}\left(2^{-}\right) S^{(0)}\left(\hat{1}^{+}\right),
$$

with the two contributions arising from figure 1 and 2 , respectively. The situation however is less trivial than in the case where the two soft gluons had the same helicity, and the double-soft factor is not the product of two single-soft factors.

Now, following the steps for the case of $\left\{1^{+}, 2^{+}\right\}$gluons, we can derive the subleading corrections to the double-soft function. However, unlike the previous case here we will have to take into account the contribution from both the BCFW diagrams 1 and 2 .

$$
\begin{aligned}
& \operatorname{DSL}^{(1)}\left(n+2,1^{+}, 2^{-}, 3\right) \\
& \begin{array}{c}
=\frac{[3 n+2]\langle n+22\rangle^{3}}{\langle n+21\rangle\langle 12\rangle\left\langle n+2\left|q_{12}\right| 3\right]\left(2 p_{n+2} \cdot q_{12}\right)}\left(\frac{-\left(2 p_{n+2} \cdot q_{12}\right)}{[3 n+2]\langle n+22\rangle} \lambda_{2}^{\alpha} \frac{\partial}{\partial \lambda_{3}^{\alpha}}\right. \\
\left.\quad+\frac{\left\langle n+2\left|q_{12}\right| 3\right]}{[3 n+2]\langle n+22\rangle} \lambda_{2}^{\alpha} \frac{\partial}{\partial \lambda_{n+2}^{\alpha}}-\frac{\langle 12\rangle}{\langle n+22\rangle} \tilde{\lambda}_{1}^{\dot{\alpha}} \frac{\partial}{\partial \tilde{\lambda}_{n}^{\dot{\alpha}}}\right) \\
+\frac{\langle n+23\rangle[13]^{3}}{[32][21]\left\langle n+2\left|q_{12}\right| 3\right]\left(2 p_{3} \cdot q_{12}\right)}\left(\frac{-\left(2 p_{3} \cdot q_{12}\right)}{[13]\langle n+23\rangle} \tilde{\lambda}_{1}^{\dot{\alpha}} \frac{\partial}{\partial \tilde{\lambda}_{n+2}^{\dot{\alpha}}}\right. \\
\left.\quad+\frac{\left\langle n+2\left|q_{12}\right| 3\right]}{[13]\langle n+23\rangle} \tilde{\lambda}_{1}^{\dot{\alpha}} \frac{\partial}{\partial \tilde{\lambda}_{3}^{\dot{\alpha}}}-\frac{[21]}{[13]} \lambda_{2}^{\alpha} \frac{\partial}{\partial \lambda_{3}^{\alpha}}\right) \\
\quad+\left.\operatorname{DSL}^{(1)}\left(n+2,1^{+}, 2^{-}, 3\right)\right|_{c},
\end{array}
\end{aligned}
$$

where contribution to the subleading terms coming from the contact terms, i.e. the ones with no derivative operator, and these are given by

$$
\left.\operatorname{DSL}^{(1)}\left(n+2,1^{+}, 2^{-}, 3\right)\right|_{c}=\frac{\langle n+22\rangle^{2}[1 n+2]}{\langle n+21\rangle} \frac{1}{\left(2 p_{n+2} \cdot q_{12}\right)^{2}}+\frac{[31]^{2}\langle 23\rangle}{[32]} \frac{1}{\left(2 p_{3} \cdot q_{12}\right)^{2}} .
$$

We note that the above equation can be simplified further as,

$$
\begin{aligned}
\mathrm{DSL}^{(1)}(n & \left.+2,1^{+}, 2^{-}, 3\right) \\
= & S^{(0)}\left(n+2,1^{+}, 2\right) S^{(1)}\left(n+2,2^{-}, 3\right)+S^{(0)}\left(3,2^{-}, 1\right) S^{(1)}\left(n+2,1^{+}, 3\right) \\
& +\frac{\langle 23\rangle[13]}{[32]\langle 12\rangle} \frac{1}{\left(2 p_{3} \cdot q_{12}\right)} \lambda_{2}^{\alpha} \frac{\partial}{\partial \lambda_{3}^{\alpha}}+\frac{\langle n+22\rangle[2 n+2]}{[n+21]\langle 12\rangle} \frac{1}{\left(2 p_{n+2} \cdot q_{12}\right)} \lambda_{2}^{\alpha} \frac{\partial}{\partial \lambda_{n+2}^{\alpha}} \\
& +\frac{[n+21]\langle 2 n+2\rangle}{\langle 1 n+2\rangle[21]} \frac{1}{\left(2 p_{n+2} \cdot q_{12}\right)} \tilde{\lambda}_{1}^{\dot{\alpha}} \frac{\partial}{\partial \tilde{\lambda}_{n+2}^{\dot{\alpha}}}+\frac{[31]\langle 32\rangle}{\langle 13\rangle[21]} \frac{1}{\left(2 p_{3} \cdot q_{12}\right)} \tilde{\lambda}_{1}^{\dot{\alpha}} \frac{\partial}{\partial \tilde{\lambda}_{3}^{\dot{\alpha}}} \\
& +\left.\operatorname{DSL}^{(1)}\left(n+2,1^{+}, 2^{-}, 3\right)\right|_{c} .
\end{aligned}
$$




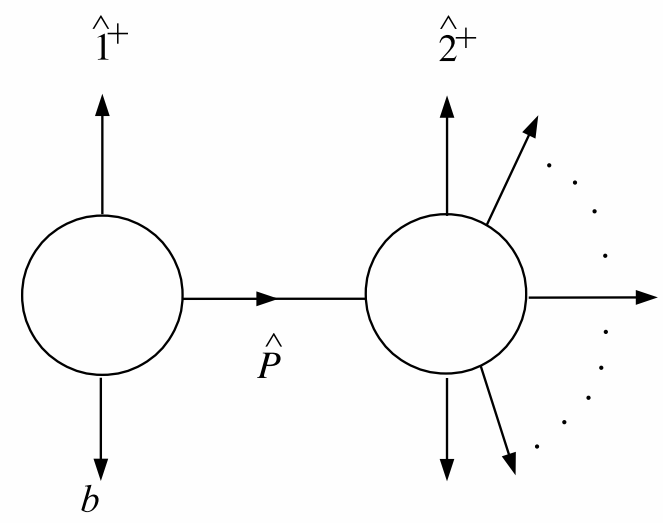

Figure 3. The first class of BCFW diagrams contributing to the double-soft factor for two gravitons. The amplitude on the left-hand side is $\overline{\mathrm{MHV}}$, and one has to sum over all possible choices of the graviton $b$.

\section{Simultaneous double-soft graviton limits}

\subsection{Summary of results}

The analysis of the double-soft limit of gravitons in terms of the BCFW recursion relations for General Relativity $[43,44]$ is entirely similar to that of gluons described in the previous section. As before, we scale the momenta of the soft particles as $q_{i} \rightarrow \delta q_{i}, i=1,2$. The main result here is that, at leading order in $\delta$ and for both choices of helicities of the gravitons becoming soft, the double-soft factor is nothing but the product of two single-soft particles (and we recall that the order in which the gravitons are taken soft is immaterial to this order, see (2.17) and (2.22)). Specifically, we define the graviton double-soft limit factor by

$$
\operatorname{DSL}\left(1^{h_{1}}, 2^{h_{2}}\right) M_{n}(3, \ldots, n+2)=\lim _{\delta \rightarrow 0} M_{n+2}\left(\delta q_{1}^{h_{1}}, \delta q_{2}^{h_{2}}, 3, \ldots, n+2\right)
$$

and find

$$
\begin{aligned}
& \operatorname{DSL}^{(0)}\left(1^{h_{1}}, 2^{h_{2}}\right)=S^{(0)}\left(1^{h_{1}}\right) S^{(0)}\left(2^{h_{2}}\right) \\
& \operatorname{DSL}^{(1)}\left(1^{h_{1}}, 2^{h_{2}}\right)=S^{(0)}\left(1^{h_{1}}\right) S^{(1)}\left(2^{h_{2}}\right)+S^{(0)}\left(2^{h_{2}}\right) S^{(1)}\left(1^{h_{1}}\right)+\left.\operatorname{DSL}^{(1)}\left(1^{h_{1}}, 2^{h_{2}}\right)\right|_{c},
\end{aligned}
$$

where $S^{(i)}\left(s^{ \pm}\right)$are the single-soft factors for graviton $s^{ \pm}$given in (2.4). The contact term at subleading order, $\left.\mathrm{DSL}^{(1)}\left(1^{h_{1}}, 2^{h_{2}}\right)\right|_{c}$, vanishes for identical helicities $h_{1}=h_{2}$ of the soft gravitons and takes the form

$$
\left.\operatorname{DSL}^{(1)}\left(1^{+}, 2^{-}\right)\right|_{c}=\frac{1}{q_{12}^{2}} \sum_{a \neq 1,2} \frac{[1 a]^{3}\langle 2 a\rangle^{3}}{\langle 1 a\rangle[2 a]} \frac{1}{2 p_{a} \cdot q_{12}},
$$

in the mixed helicity case. Note that both double-soft factors diverge at leading order as $1 / \delta^{2}$. Differences to the consecutive soft-limit appear only in the contact term at subleading order $1 / \delta$ in the mixed helicity case. 


\subsection{Derivation from the BCFW recursion relation}

As for the case of gluons, we distinguish two cases depending on whether the two gravitons becoming soft have the same or opposite helicities. We outline below the main steps of the derivations.

The $\mathbf{1}^{+} \mathbf{2}^{+}$case. The first relevant class of diagram is shown in figure 3 , where $b$ can be any of the $n$ hard particles. For the sake of definiteness we illustrate the case where $b$ has positive helicity; the case where $b$ has negative helicity leads to an identical result. Using the fact that the momentum $\hat{q}_{2}$ is becoming soft we can write this diagram as

$$
M_{3}\left(b^{+}, \hat{1}^{+}, \hat{P}^{-}\right) \frac{1}{\left(q_{1}+p_{b}\right)^{2}} M_{n}\left(\hat{2}^{+}, \hat{P}, \ldots\right)
$$

where $S^{(0)}\left(s^{+}\right)$is given in (2.4), and $x$ and $y$ denote two arbitrary reference spinors. Using the explicit expression for the three-point anti-MHV amplitude and the shifts derived earlier, and that $\hat{P}=p_{b}+\delta \frac{\langle 1 b\rangle}{\langle 2 b\rangle}|b\rangle[1 \mid$ we may rewrite the last term in the above with the soft shifted leg $\hat{2}$ as

$M_{n}\left(\hat{2}^{+}, p_{b}+\delta \frac{\langle 1 b\rangle}{\langle 2 b\rangle}|b\rangle[1 \mid, \ldots)=e^{\delta \frac{\langle 1 b\rangle}{2 b b\rangle}\left[1 \partial_{b}\right]}\left(\frac{1}{\delta} S^{(0)}\left(\hat{2}^{+}\right)+S^{(1)}\left(\hat{2}^{+}\right)+\delta S^{(2)}\left(\hat{2}^{+}\right)\right) M_{n}(b, \ldots)\right.$.

From this expressions all relevant leading and subleading contributions to the simultaneous soft factor may be extracted:

$$
\operatorname{DSL}\left(1^{+}, 2^{+}\right)=\frac{M_{3}\left(b^{+}, \hat{1}^{+}, \hat{P}^{-}\right)}{\left(q_{1}+p_{b}\right)^{2}} e^{\delta \frac{\langle 1 b\rangle}{\langle 2 b\rangle}\left[1 \partial_{b}\right]}\left(\frac{1}{\delta} S^{(0)}\left(\hat{2}^{+}\right)+S^{(1)}\left(\hat{2}^{+}\right)+\delta S^{(2)}\left(\hat{2}^{+}\right)\right) .
$$

At leading order we find

$$
\operatorname{DSL}^{(0)}\left(1^{+}, 2^{+}\right) M_{n}(b, \ldots)
$$

with

$$
\begin{aligned}
\operatorname{DSL}^{(0)}\left(1^{+}, 2^{+}\right) & =\frac{1}{\langle 12\rangle^{2}} \sum_{b \neq 1,2} \frac{[b 1]\langle b 2\rangle^{2}}{\langle 1 b\rangle} S^{(0)}(\hat{2}) \\
& =\frac{1}{\langle 12\rangle^{2}} \sum_{a, b \neq 1,2} \frac{[b 1]\langle b 2\rangle}{\langle 1 b\rangle} \frac{\left\langle b\left|q_{12}\right| a\right]}{\langle 2 a\rangle} \frac{\langle x a\rangle\langle y a\rangle}{\langle x 2\rangle\langle y 2\rangle} .
\end{aligned}
$$

The expression (4.9) is symmetric in the two soft particles, 1 and 2, although not manifestly. Furthermore, it turns out using total momentum conservation that

$$
\operatorname{DSL}^{(0)}\left(1^{+}, 2^{+}\right)=S^{(0)}\left(1^{+}\right) S^{(0)}\left(2^{+}\right),
$$

i.e. the double-soft factor for gravitons with the same helicity is the product of two singlesoft factors. Again it is not a local expression, in the sense explained in section 3. 


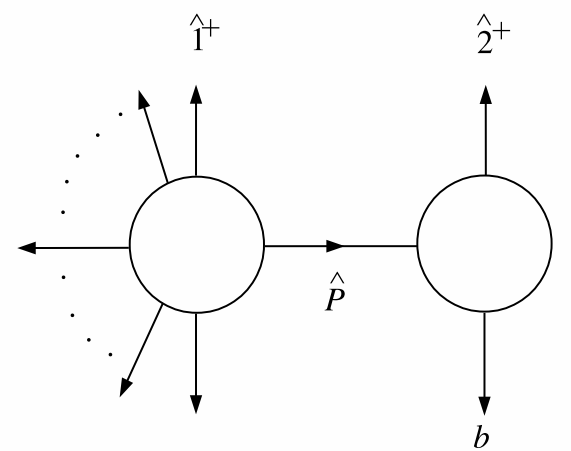

Figure 4. The second class of BCFW diagram contributing to the double-soft graviton factor. The three-point amplitude is MHV and one has to sum over all possible choices of graviton $b$. Similarly to the gluon case, this diagram contributes only when graviton 2 has negative helicity.

One can also work out the first subleading contribution to the double-soft limit. The result reads for the non-contact term

$$
\begin{gathered}
\left.\operatorname{DSL}^{(1)}\left(1^{+}, 2^{+}\right)\right|_{\mathrm{nc}} \\
\begin{aligned}
\frac{1}{\langle 12\rangle^{2}} \sum_{a, b \neq 1,2} \frac{[b 1]\langle b 2\rangle}{\langle 1 b\rangle} \frac{\left\langle b\left|q_{12}\right| a\right]}{\langle 2 a\rangle}\left[\frac{1}{2}\left(\frac{\langle x a\rangle}{\langle x 2\rangle}+\frac{\langle y a\rangle}{\langle y 2\rangle}\right)\left(\tilde{\lambda}_{2}^{\dot{\alpha}} \frac{\partial}{\partial \tilde{\lambda}_{a}^{\dot{\alpha}}}+\frac{\langle 1 b\rangle}{\langle 2 b\rangle} \tilde{\lambda}_{1}^{\dot{\alpha}} \frac{\partial}{\partial \tilde{\lambda}_{a}^{\dot{\alpha}}}\right)\right. \\
\left.+\frac{\langle x a\rangle\langle y a\rangle\langle 12\rangle}{\langle x 2\rangle\langle y 2\rangle\langle b 2\rangle} \tilde{\lambda}_{1}^{\dot{\alpha}} \frac{\partial}{\partial \tilde{\lambda}_{b}^{\dot{\alpha}}}\right]
\end{aligned}
\end{gathered}
$$

Making the gauge choice $\lambda_{x}=\lambda_{y}=\lambda_{1}$ to make contact to the discussion in section 2.2 we find

$$
\begin{aligned}
\left.\operatorname{DSL}^{(1)}\left(1^{+}, 2^{+}\right)\right|_{\mathrm{nc}}=\frac{1}{\langle 12\rangle^{3}} & \sum_{a, b \neq 1,2} \frac{[b 1]\langle b 2\rangle}{\langle 1 b\rangle} \frac{\left\langle b\left|q_{12}\right| a\right]\langle 1 a\rangle}{\langle 2 a\rangle} \\
& \times\left[\tilde{\lambda}_{2}^{\dot{\alpha}} \frac{\partial}{\partial \tilde{\lambda}_{a}^{\dot{\alpha}}}+\frac{\langle 1 b\rangle}{\langle 2 b\rangle} \tilde{\lambda}_{1}^{\dot{\alpha}} \frac{\partial}{\partial \tilde{\lambda}_{a}^{\dot{\alpha}}}-\frac{\langle 1 a\rangle}{\langle 2 b\rangle} \tilde{\lambda}_{1}^{\dot{\alpha}} \frac{\partial}{\partial \tilde{\lambda}_{b}^{\dot{\alpha}}}\right] .
\end{aligned}
$$

In fact the middle term vanishes by momentum conservation $\left.\sum_{b} \mid b\right]\langle b|=0$. The structure may be further reduced by splitting up the $\left\langle b\left|q_{1}+q_{2}\right| a\right]$ factor and using momentum conservation and the Lorentz invariance $\sum_{b}[1 b]\left[1 \tilde{\partial}_{b}\right] \mathcal{A}=0$. This lets us rewrite this double-soft factor as

$$
\left.\mathrm{DSL}^{(1)}\left(1^{+}, 2^{+}\right)\right|_{\mathrm{nc}}=S^{(0)}\left(1^{+}\right) S^{(1)}\left(2^{+}\right)+S^{(0)}\left(2^{+}\right) S^{(1)}\left(1^{+}\right) .
$$

We also get a contact term contribution to the above subleading factor when the derivative operator $\left[1 \partial_{b}\right]$ in the exponential in (4.7) hits the leading soft function $S^{(0)}\left(\hat{2}^{+}\right)$,

$$
\left.\operatorname{DSL}^{(1)}\left(1^{+}, 2^{+}\right)\right|_{c}=\frac{[12]}{\langle 12\rangle^{3}}\left\langle 1\left|\sum_{b \neq 1,2} p_{b}\right| 1\right]=0 .
$$

As for the case of soft gluons, we have to consider another diagram which is however vanishing as we take the two particles soft. This diagram is depicted in figure 4. A short 
calculation shows that the contribution of this diagram is at the leading order in $\delta$

$$
\left(\frac{\langle\hat{P} 3\rangle^{3}}{\langle\hat{P} 2\rangle\langle 23\rangle}\right)^{2} \frac{1}{\langle 2 b\rangle[b 2]} S^{(0)}\left(\hat{1}^{+}\right)=\frac{[12]^{6}}{[13]^{2}[23]^{2}} S^{(0)}\left(\hat{1}^{+}\right),
$$

times an $n$-point amplitude. This quantity is immediately seen to vanish as we take the momenta of particles 1 and 2 soft and thus irrelevant at the first three leading orders. Similarly, one also convinces oneself that the generic BCFW diagram with $n>3$ point amplitudes to the right or left is finite in the soft limit and therefore not contributing to the considered leading orders. As soon as diagrams of this type start contributing the universality is lost and there is no double-soft factor.

The $\mathbf{1}^{+} \mathbf{2}^{-}$case. The analysis of this case proceeds in a very similar way as for gluons. Again there are two diagrams contributing, depicted in figures 3 and 4 . The calculations of these diagrams is straightforward and involves the soft factors $S\left(\hat{2}^{-}\right)$and $S\left(\hat{1}^{+}\right)$, respectively. These soft factors are given by, ${ }^{5}$

$$
\begin{array}{ll}
S^{(0)}\left(\hat{2}^{-}\right)=\sum_{a \neq 1,2} \frac{\langle 2 a\rangle[x a][y a]}{[\hat{2} a][x \hat{2}][y \hat{2}]}, & S^{(1)}\left(\hat{2}^{-}\right)=\frac{1}{2} \sum_{a \neq 1,2} \frac{\langle 2 a\rangle}{[\hat{2} a]}\left(\frac{[x a]}{[x \hat{2}]}+\frac{[y a]}{[y \hat{2}]}\right)\left\langle 2 \partial_{a}\right\rangle \\
S^{(0)}\left(\hat{1}^{+}\right)=\sum_{a \neq 1,2} \frac{[1 a]\langle x a\rangle\langle y a\rangle}{\langle\hat{1} a\rangle\langle x \hat{1}\rangle\langle y \hat{1}\rangle}, & S^{(1)}\left(\hat{1}^{+}\right)=\frac{1}{2} \sum_{a \neq 1,2} \frac{[1 a]}{\langle\hat{1} a\rangle}\left(\frac{\langle x a\rangle}{\langle x \hat{1}\rangle}+\frac{\langle y a\rangle}{\langle y \hat{1}\rangle}\right)\left[1 \partial_{a}\right]
\end{array}
$$

where

$$
\hat{\tilde{\lambda}}_{2}=\frac{q_{12}|b\rangle}{\langle 2 b\rangle}
$$

for the first recursive diagram, and

$$
\hat{\lambda}_{1}=\frac{\left.q_{12} \mid b\right]}{[1 b]}
$$

for the second one. It is particularly convenient to choose $\tilde{\lambda}_{x}=\tilde{\lambda}_{y}=\tilde{\lambda}_{1}$ and $\lambda_{x}=\lambda_{y}=\lambda_{2}$, for the first and second diagram, respectively. Doing so, we obtain from the first diagram

$$
\frac{1}{\delta} \frac{\langle 2 b\rangle^{2}[b 1]}{\langle 12\rangle^{2}\langle 1 b\rangle} e^{\delta \frac{\langle 12\rangle}{\langle b 2\rangle}\left[1 \partial_{b}\right]}\left\{\frac{1}{\delta} S^{(0)}\left(\hat{2}^{-}\right)+S^{(1)}\left(\hat{2}^{-}\right)\right\} M_{n}(3, \ldots, n+2),
$$

while, for the second,

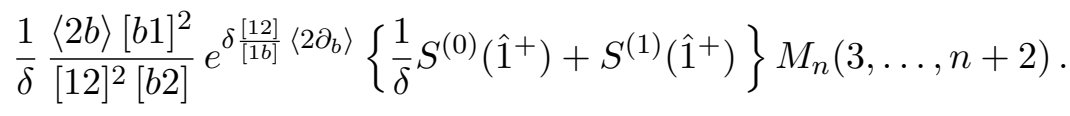

The double-soft factor for soft gravitons $1^{+} 2^{-}$is obtained by summing the two contributions in (4.20) and (4.21). At leading order we find

$$
\operatorname{DSL}^{(0)}\left(1^{+}, 2^{-}\right)=\frac{1}{q_{12}^{4}} \sum_{a, b \neq 1,2}\left[\frac{\langle 2 b\rangle^{3}[1 a]^{2}[1 b]\langle 2 a\rangle}{\langle 1 b\rangle\left\langle b\left|q_{12}\right| a\right]}+\frac{[1 b]^{3}\langle 2 a\rangle^{2}\langle 2 b\rangle[1 a]}{[2 b]\left[b\left|q_{12}\right| a\right\rangle}\right] .
$$

\footnotetext{
${ }^{5}$ Recall that we are using a $\langle 12]$ shift, which explains the various hatted quantities in (4.16) and (4.17).
} 
In fact, we can easily combine the two terms in (4.22) and show that we just get the result of the consecutive limit discussed earlier in (2.21). To this end, in the second term in (4.22) we relabel $a \leftrightarrow b$ and use

$$
\frac{\langle 2 b\rangle}{\langle 1 b\rangle}+\frac{[1 a]}{[2 a]}=-\frac{\left[a\left|q_{12}\right| b\right\rangle}{\langle 1 b\rangle[2 a]} .
$$

Hence we conclude that

$$
\operatorname{DSL}^{(0)}\left(1^{+}, 2^{-}\right)=S^{(0)}\left(1^{+}\right) S^{(0)}\left(2^{-}\right) .
$$

Working out the first subleading contribution to the double-soft limit for the mixed helicity assignments from (4.20) and (4.21) one finds for the non-contact terms

$$
\begin{aligned}
\left.\operatorname{DSL}^{(1)}\left(1^{+}, 2^{-}\right)\right|_{\mathrm{nc}} & =\frac{1}{q_{12}^{4}} \sum_{a, b \neq 1,2} \frac{[1 a]^{2}[1 b]\langle 2 a\rangle\langle 2 b\rangle^{2}}{\langle b 1\rangle[2 a]}\left(\frac{[12]}{[1 a]} \lambda_{2}^{\alpha} \frac{\partial}{\partial \lambda_{a}^{\alpha}}-\frac{\langle 12\rangle}{\langle 2 b\rangle} \tilde{\lambda}_{1}^{\dot{\alpha}} \frac{\partial}{\partial \tilde{\lambda}_{b}^{\dot{\alpha}}}\right) \\
& =S^{(0)}\left(1^{+}\right) S^{(1)}\left(2^{-}\right)+S^{(0)}\left(2^{-}\right) S^{(1)}\left(1^{+}\right) .
\end{aligned}
$$

where the same gauge choices for the reference spinors as above were made. This subleading term also has a contribution from contact terms given by

$$
\begin{aligned}
\left.\operatorname{DSL}^{(1)}\left(1^{+}, 2^{-}\right)\right|_{c} & =\frac{1}{q_{12}^{2}} \sum_{b \neq 1,2}\left(\frac{[1 b]^{4}\langle 2 b\rangle^{3}}{[b 2]\left(2 p_{b} \cdot q_{12}\right)^{2}}+\frac{[1 b]^{3}\langle 2 b\rangle^{4}}{\langle b 1\rangle\left(2 p_{b} \cdot q_{12}\right)^{2}}\right) \\
& =\frac{1}{q_{12}^{2}} \sum_{b \neq 1,2} \frac{[1 b]^{3}\langle 2 b\rangle^{3}}{[2 b]\langle 1 b\rangle} \frac{1}{2 p_{b} \cdot q_{12}} .
\end{aligned}
$$

We hence see, that a difference to the consecutive double-soft limit appears at the subleading order in the contact term above, cf. (2.26).

\section{Double-soft scalars in $\mathcal{N}=4$ super Yang-Mills}

The emission of a single soft scalar in $\mathcal{N}=4$ super Yang-Mills does not lead to any divergence - the amplitude after a soft scalar has been emitted is in general finite. Thus, the consecutive limit where two scalars are taken soft is also finite and not universal. It is then interesting that the simultaneous double-soft scalar limit does lead to a universal divergent structure, which can also be analysed using recursion relations.

To begin it is useful to look at simple examples. We take two scalars in a singlet configuration, and consider the amplitudes $A\left(1_{\phi_{12}}, 2_{\phi_{34}}, g_{3}, g_{4}, g_{5}\right)$, where the helicities of the gluons $\left(g_{3}, g_{4}, g_{5}\right)$ are a permutation of $(--+)$. It is then easy to extract the doublesoft limit:

$$
A\left(1_{\phi_{12}}, 2_{\phi_{34}}, g_{3}, g_{4}, g_{5}\right) \rightarrow \frac{[23][15]\langle 53\rangle}{s_{125} s_{123}[12]} A\left(g_{3}, g_{4}, g_{5}\right) .
$$

Note that the prefactor appearing in this equation is divergent in the double-soft limit. In the following we wish to derive such kind of behaviour from a recursion relation. One direct approach is to perform the supersymmetric generalisation of the $\langle 12]$-shift used in previous sections:

$$
\hat{\lambda}_{1}:=\lambda_{1}+z \lambda_{2}, \quad \hat{\tilde{\lambda}}_{2}:=\tilde{\lambda}_{2}-z \tilde{\lambda}_{1}, \quad \hat{\eta}_{2}=\eta_{2}-z \eta_{1} .
$$


As in the bosonic case there are two special BCFW diagrams to consider: figure 1, where the three-point amplitude sits on the left with the external legs $\hat{1}$ and $n+2$ and figure 2 with the three-point amplitude on the right-hand side with external legs $\hat{2}$ and 3 (where now particles 1 and 2 are scalars). If we take the holomorphic limit discussed in appendix B for both particle 1 and 2 we will find the supersymmetric generalisation of the bosonic $1^{+} 2^{+}$case. Instead we will consider taking the holomorphic limit of particle 1 and the antiholomorphic limit of particle 2 which is the supersymmetric generalisation of the $1^{+} 2^{-}$ case; as in that case we find contributions from both BCFW diagrams. The calculation is essentialy identical to the bosonic case and so we will omit the details. The contribution from figure 1 is

$$
\int d^{4} \eta_{P} A_{3}^{\overline{\mathrm{MHV}}}(n+2, \hat{1}, \hat{P}) \frac{1}{\langle 1 n+2\rangle[n+21]} \bar{S}(-\hat{P}, \hat{2}, 3) A_{n}(-\hat{P}, 3, \ldots),
$$

where $A_{3}^{\overline{\mathrm{MHV}}}$ is the supersymmetric $\overline{\mathrm{MHV}}$ three-point amplitude and $\bar{S}(a, s, b)$ is the antiholomorphic soft factor described in appendix B. Performing the integrations over the internal Graßmann parameters we can extract the contribution to the appropriate doublesoft factor by examining the coefficient of the relevant $\eta$ 's. For particle 1 and 2 being scalars in the singlet state, i.e. the coefficient of the $\eta_{1}^{2} \eta_{2}^{2}$ term, the leading order contribution is

$$
\operatorname{DSL}_{\mathrm{a}}\left(n+2,1_{\phi}, 2_{\phi}, 3\right)=\frac{\langle n+22\rangle[n+23]\langle n+21\rangle}{2 p_{n+2} \cdot q_{12}\langle 12\rangle\left\langle n+2\left|q_{12}\right| 3\right]} .
$$

The contribution from figure 2 is

$$
\int d \eta_{P} S(n+2, \hat{1}, \hat{P}) A_{n}(n+2, \hat{P}, \ldots) \frac{1}{p_{23}^{2}} A_{3}^{\mathrm{MHV}}(\hat{2}, 3,-\hat{P}),
$$

where now $S(a, s, b)$ is the holomorphic factor in appendix B. This diagram contributes to the singlet scalar double-soft coefficient the term

$$
\operatorname{DSL}_{\mathrm{b}}\left(n+2,1_{\phi}, 2_{\phi}, 3\right)=-\frac{\langle n+23\rangle[31][32]}{2 p_{3} \cdot q_{12}\left\langle n+2\left|q_{12}\right| 3\right][12]} .
$$

To find the complete double soft factor we combine the two terms i.e.

$$
\operatorname{DSL}\left(n+2,1_{\phi}, 2_{\phi}, 3\right)=\operatorname{DSL}_{\mathrm{a}}\left(n+2,1_{\phi}, 2_{\phi}, 3\right)+\mathrm{DSL}_{\mathrm{b}}\left(n+2,1_{\phi}, 2_{\phi}, 3\right) .
$$

For the sake of illustration, we derive the result (5.1) for the particular case of $\left(g_{3}, g_{4}, g_{5}\right)=$ $\left(3^{-}, 4^{-}, 5^{+}\right)$, with the scalars in a flavour singlet configuration. Due to the three-particle kinematics we have

$$
\tilde{\lambda}_{3} \propto \tilde{\lambda}_{4} \propto \tilde{\lambda}_{5}
$$

and hence for this particular choice the contribution from $\mathrm{DSL}_{\mathrm{a}}$ is zero. Moreover we can exchange $\mid 5]$ and $\mid 3]$ in the expression $\mathrm{DSL}_{\mathrm{b}}$ as the constants of proportionality cancel between the numerator and denominator, hence

$$
\operatorname{DSL}_{\mathrm{b}}\left(5,1_{\phi}, 2_{\phi}, 3\right)=-\frac{\langle 53\rangle[31][32]}{\left\langle 3\left|q_{12}\right| 3\right][12]\left\langle 5\left|q_{12}\right| 3\right]}=\frac{\langle 53\rangle[51][23]}{\left\langle 3\left|q_{12}\right| 3\right][12]\left\langle 5\left|q_{12}\right| 5\right]},
$$

in agreement with (5.1) at leading order in the double-soft expansion. 


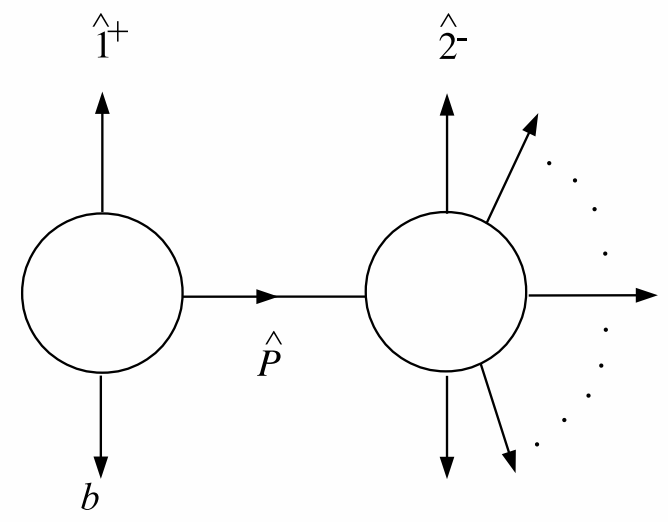

Figure 5. The first BCFW diagram contributing to the double-soft scalar limit.

We can also re-derive this result from a different recursion relation, where we shift one of the two soft particles and one hard particle. Taking again the scalars in positions 1 and 2 , we shift one of the scalars, say 2 , and an adjacent hard particle 3 ,

$$
\lambda_{\hat{2}}=\lambda_{2}+z \lambda_{3}, \quad \tilde{\lambda}_{\hat{3}}=\tilde{\lambda}_{3}-z \tilde{\lambda}_{2}, \quad \eta_{\hat{3}}=\eta_{3}-z \eta_{2} .
$$

There are two recursion diagrams to consider, shown in figures 5 and 6 . We begin discussing the first one, where we have a four-point amplitude with both soft legs attached to it. To leading order in the soft parameter $\delta$, the position of the pole in $z$ is

$$
z_{*}=\frac{2 p_{n} \cdot q_{12}}{\langle 3 n+2\rangle[2 n+2]} .
$$

The BCFW diagram in figure 5 is then

$$
A_{n+2}=\int d^{4} \eta_{\hat{P}} A_{4}(n+2,1, \hat{2}, \hat{P}) \frac{1}{P^{2}} A_{n}(-\hat{P}, \hat{3}, \ldots),
$$

where $P^{2}=\left(q_{12}+p_{n+2}\right)^{2} \simeq 2 q_{12} \cdot p_{n+2}$, and the four-point superamplitude is explicitly given by

$$
A_{4}(1, \hat{2}, \hat{P}, n+2)=\frac{\delta^{(8)}\left(\lambda_{1} \eta_{1}+\lambda_{\hat{2}} \eta_{2}+\lambda_{\hat{P}} \eta_{\hat{P}}+\lambda_{n+2} \eta_{n+2}\right)}{\langle 1 \hat{2}\rangle\langle\hat{2} \hat{P}\rangle\langle\hat{P} n+2\rangle\langle n+21\rangle} .
$$

We can re-write the fermionic delta function as

$$
\begin{aligned}
\delta^{(8)}\left(\lambda_{1} \eta_{1}+\lambda_{\hat{2}} \eta_{2}+\lambda_{\hat{P}} \eta_{\hat{P}}+\lambda_{n+2} \eta_{n+2}\right)= & \langle\hat{2} \hat{P}\rangle^{4} \delta^{(4)}\left(\eta_{\hat{P}}+\eta_{1} \frac{\langle 1 \hat{2}\rangle}{\langle\hat{P} \hat{2}\rangle}+\eta_{n+2} \frac{\langle n+2 \hat{2}\rangle}{\langle\hat{P} \hat{2}\rangle}\right) \\
& \times \delta^{(4)}\left(\eta_{2}+\eta_{1} \frac{\langle 1 \hat{P}\rangle}{\langle\hat{2} \hat{P}\rangle}+\eta_{n+2} \frac{\langle n+2 \hat{P}\rangle}{\langle\hat{2} \hat{P}\rangle}\right), \quad(5
\end{aligned}
$$

thus getting

$$
\frac{\langle\hat{2} \hat{P}\rangle^{3}}{\langle 1 \hat{2}\rangle\langle\hat{P} n+2\rangle\langle n+21\rangle} \delta^{(4)}\left(\eta_{2}+\eta_{1} \frac{\langle 1 \hat{P}\rangle}{\langle\hat{2} \hat{P}\rangle}+\eta_{n+2} \frac{\langle n+2 \hat{P}\rangle}{\langle\hat{2} \hat{P}\rangle}\right) A_{n}(-\hat{P}, \hat{3}, \ldots, n+1),
$$




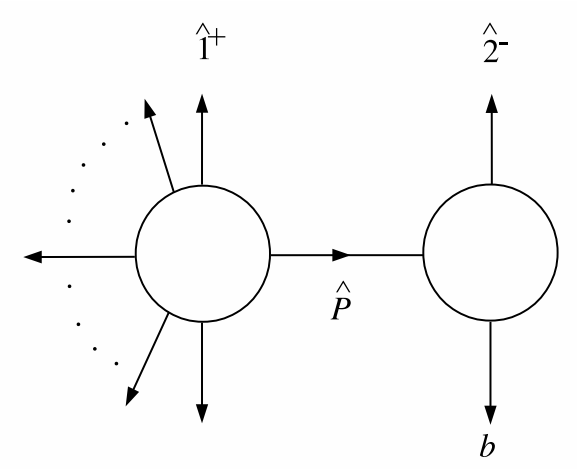

Figure 6. The second BCFW diagram contributing to the double-soft scalar limit. This diagram does not contribute when the two scalars are in a flavour non-singlet configuration.

where now $A_{n}$ is evaluated at

$$
\eta_{\hat{P}}=-\eta_{1} \frac{\langle 1 \hat{2}\rangle}{\langle\hat{P} \hat{2}\rangle}-\eta_{n+2} \frac{\langle n+2 \hat{2}\rangle}{\langle\hat{P} \hat{2}\rangle} .
$$

One can also easily work out ${ }^{6}$

$$
\begin{aligned}
\langle 1 \hat{P}\rangle & \sim\langle 1 n+2\rangle, & \langle\hat{2} \hat{P}\rangle & \sim \frac{\langle 1 n+2\rangle[n+21]}{[n+22]}, \\
\langle\hat{P} n+2\rangle & \sim \frac{[12]\langle 1 n+2\rangle}{[n+22]}, & \langle 1 \hat{2}\rangle & =\frac{\langle n+21\rangle\left\langle 3\left|q_{12}\right| n+2\right]}{\langle 3 n+2\rangle[n+22]},
\end{aligned}
$$

so that (5.15) becomes

$$
\frac{[n+21]^{3}\langle 3 n+2\rangle}{[n+22][12]\left\langle 3\left|q_{12}\right| n+2\right]} \delta^{(4)}\left(\eta_{2}+\eta_{1} \frac{\langle 1 \hat{P}\rangle}{\langle\hat{2} \hat{P}\rangle}+\eta_{n+2} \frac{\langle n+2 \hat{P}\rangle}{\langle\hat{2} \hat{P}\rangle}\right) A_{n}(-\hat{P}, \hat{3}, \ldots, n+1) .
$$

The second diagram is easily seen to contribute

$$
\begin{aligned}
\frac{\langle 13\rangle}{\langle 12\rangle\langle 23\rangle} A_{n+1}\left(\left\{-\lambda_{1}, \tilde{\lambda}_{1}+\right.\right. & \left.\tilde{\lambda}_{2} \frac{\langle 23\rangle}{\langle 13\rangle}, \eta_{1}+\eta_{2} \frac{\langle 23\rangle}{\langle 13\rangle}\right\}, \\
& \left.\left\{\lambda_{3}, \tilde{\lambda}_{3}+\lambda_{2} \frac{\langle 12\rangle}{\langle 13\rangle}, \eta_{3}+\frac{\langle 12\rangle}{\langle 13\rangle} \eta_{2}\right\},\{4\} \ldots,\{n+2\}\right),
\end{aligned}
$$

where we notice that the prefactor is divergent only if we simultaneously make the momenta $q_{1}$ and $q_{2}$ soft.

At this point we have to take components of (the sum of) (5.18) and (5.19). One can distinguish two basic cases, namely whether the two scalars are in a singlet or non-singlet helicity configuration. In the latter case, only the recursion diagram in figure 5, given by (5.18), contributes. For the sake of illustration, we derive the result (5.1) for the particular case of $\left(g_{3}, g_{4}, g_{5}\right)=\left(3^{-}, 4^{-}, 5^{+}\right)$, with the scalars in a flavour singlet configuration. For this particular choice, the diagram in figure 6 vanishes since the amplitude on the

\footnotetext{
${ }^{6}$ The $\sim$ sign means that an equality holds at leading order in the double-soft limit.
} 
left-hand side would have to be MHV, and thus vanishing given our choice of shifts. One is then left with the contribution from figure 5 , which is equal to

$$
\frac{[51][52]\langle 35\rangle}{\langle 34\rangle[34][12]\left\langle 3\left|q_{12}\right| 5\right]} A_{3}\left(3^{-}, 4^{-}, 5^{+}\right),
$$

in agreement with (5.1) at leading order in the double-soft expansion.

Next we discuss another particularly simple situation, where particle 3 is a negativehelicity gluon, and we take the two scalars in a non-singlet flavour configuration. In this case the diagram of figure 6 does not contribute and furthermore there is only one way to extract a contribution from the diagram in figure 5. Specifically, we take two powers of $\eta_{2}$ and only one power of $\eta_{1}$ from the $\delta^{(4)}$ in (5.15), while the remaining power of $\eta_{1}$ will come from differentiating the amplitude on the right-hand side of the recursion. Doing so we get

$$
\begin{aligned}
\frac{\langle\hat{2} \hat{P}\rangle^{3}}{\langle 1 \hat{2}\rangle\langle\hat{P} n+2\rangle\langle n+21\rangle}\left(\frac{\langle 1 \hat{P}\rangle}{\langle\hat{2} \hat{P}\rangle}\right)\left(\frac{\langle n+2 \hat{P}\rangle}{\langle\hat{2} \hat{P}\rangle}\right)\left(\frac{\langle 1 \hat{2}\rangle}{\langle\hat{2} \hat{P}\rangle}\right) \\
\cdot \epsilon_{a_{1} a_{2} a_{3} a_{4}} \eta_{2}^{a_{1}} \eta_{2}^{a_{2}} \eta_{1}^{a_{3}} \eta_{n+2}^{a_{4}} \eta_{1}^{a_{5}} \frac{\partial}{\partial \eta_{\hat{P}}^{a_{5}}} A_{n}(-\hat{P}, \hat{3}, \ldots n+1),
\end{aligned}
$$

which after using (5.17) becomes simply

$$
A_{n+2} \rightarrow \frac{1}{p_{n+2} \cdot q_{12}} \epsilon_{a_{1} a_{2} a_{3} a_{4}} \eta_{2}^{a_{1}} \eta_{2}^{a_{2}} \eta_{1}^{a_{3}} \eta_{n+2}^{a_{4}} \eta_{1}^{a_{5}} \frac{\partial}{\partial \eta_{\hat{P}}^{a_{5}}} A_{n}\left(-\hat{P}, g_{3}^{-}, \ldots n+1\right)
$$

where we recall that we selected particle 3 to be a gluon of negative helicity. This contribution diverges as $1 / \delta$ in the double-soft limit. We also note that this case is entirely similar to that discussed in [31] (however note that in that case, particle 3 was replaced by an auxiliary negative-helicity graviton, which was taken soft and decoupled at the end of the calculation).

\section{Acknowledgments}

We would like to thank Lorenzo Bianchi, Massimo Bianchi, Andi Brandhuber, Ed Hughes, Bill Spence and Congkao Wen for discussions on related topics. GT thanks the Institute for Physics, IRIS Adlershof and the Kolleg Mathematik und Physik at Humboldt University, Berlin, as well as the Physics Department at the University of Rome "Tor Vergata" for their warm hospitality and support. The work of GT was supported by the Science and Technology Facilities Council Consolidated Grant ST/L000415/1 "String theory, gauge theory $\&$ duality". The work of TMcL was supported in part by Marie Curie Grant CIG333851. DN's research is supported by the SFB 647 "Raum-Zeit-Materie. Analytische und Geometrische Strukturen" grant.

\section{A Sub-subleading terms}

We can continue our analysis of the double-soft terms in the gravitational case to the sub-subleading terms. For the consecutive double-soft limit we have we have

$$
\mathrm{CSL}^{(2)}\left(1^{+}, 2^{ \pm}\right)=S^{(1)}\left(q_{2}^{ \pm}\right) S^{(1)}\left(q_{1}^{+}\right)+S^{(0)}\left(q_{2}^{ \pm}\right) S^{(2)}\left(q_{1}^{+}\right)+S^{(2)}\left(q_{2}^{ \pm}\right) S^{(1)}\left(q_{1}^{+}\right) .
$$


The $1^{+} 2^{+}$case. A brief calculation shows that in the case of two positive helicity gluons

$$
\begin{aligned}
\operatorname{CSL}^{(2)}\left(1^{+}, 2^{+}\right)= & -\frac{[12]}{\langle 12\rangle^{2}} \sum_{a \neq 1,2}\left\langle a\left|q_{12}\right| a\right] \frac{\left[2 \partial_{\tilde{a}}\right]}{\langle 1 a\rangle} \\
& +\frac{1}{2\langle 12\rangle^{2}} \sum_{a, b \neq 1,2} \frac{[2 a][1 b]}{\langle 2 a\rangle\langle 1 b\rangle}\left(\langle 1 a\rangle\left[1 \partial_{\tilde{b}}\right]-\langle 2 b\rangle\left[2 \partial_{\tilde{a}}\right]\right)^{2}
\end{aligned}
$$

where we have used the notation $\left[1 \partial_{\tilde{a}}\right]=\tilde{\lambda}_{1}^{\dot{\alpha}} \frac{\partial}{\partial \tilde{\lambda}_{a}^{\dot{\alpha}}}$ etc. Because of the contact term the antisymmetric combination is non-trivial and can be simplified to

$$
\operatorname{aCSL}^{(2)}\left(1^{+}, 2^{+}\right)=-\frac{[12]}{2\langle 12\rangle^{2}} \sum_{a \neq 1,2}\left(\frac{\langle 1 a\rangle}{\langle 2 a\rangle}[1 a]\left[1 \partial_{\tilde{a}}\right]-\frac{\langle 2 a\rangle}{\langle 1 a\rangle}[2 a]\left[2 \partial_{\tilde{a}}\right]\right) .
$$

The $1^{+} 2^{-}$case. For the mixed helicity case we find

$$
\begin{aligned}
\operatorname{CSL}^{(2)}\left(1^{+}, 2^{-}\right)= & \frac{1}{[12]\langle 12\rangle} \sum_{a \neq 1,2} \frac{[1 a]\langle 2 a\rangle^{4}}{\langle 1 a\rangle^{3}} \\
& +\sum_{a \neq 1,2} \frac{\langle 2 a\rangle^{2}[1 a]}{[2 a]\langle 1 a\rangle^{2}}\left(\frac{[1 a]}{[12]}\left[1 \partial_{\tilde{a}}\right]-\frac{\langle 2 a\rangle}{2\langle 21\rangle}\left\langle 2 \partial_{a}\right\rangle\right) \\
& +\frac{1}{2} \sum_{a, b \neq 1,2} \frac{\langle 2 a\rangle[1 b]}{[2 a]\langle 1 b\rangle}\left(\frac{[1 a]}{[12]}\left[1 \partial_{\tilde{b}}\right]-\frac{\langle 2 b\rangle}{\langle 21\rangle}\left\langle 2 \partial_{a}\right\rangle\right)^{2}
\end{aligned}
$$

where in the last line the expression should be understood with the derivatives always to the right, i.e. they don't act on the $\lambda / \tilde{\lambda}$ 's in the double-soft factor itself. Of particular interest is the first term which arises as a contact term but one where the derivatives act on the soft momenta and so this term in fact has scaling behaviour of the same order as $\operatorname{CSL}^{(1)}$.

\section{B Supersymmetric Yang-Mills soft limits}

It is straightforward to consider the supersymmetric generalisation of the previous calculations. Let us briefly review the single soft case in Yang-Mills. Given an $(n+1)$-point superamplitude the soft limit, with particle 1 being soft, is naturally taken as

$$
\left\{\lambda_{1}, \tilde{\lambda}_{1}, \eta_{1}\right\} \rightarrow\left\{\sqrt{\delta} \lambda_{1}, \sqrt{\delta} \tilde{\lambda}_{1}, \eta_{1}\right\}
$$

with $\delta \rightarrow 0$. In particular with this choice of scaling both $q=\sum_{i} \lambda_{i} \eta_{i}$ and $\tilde{q}=\sum_{i} \tilde{\lambda}_{i} \frac{\partial}{\partial \eta_{i}}$ scale identically. Using the little transformation of the superamplitude, this implies

$$
A_{n+1}\left(\left\{\sqrt{\delta} \lambda_{1}, \sqrt{\delta} \tilde{\lambda}_{1}, \eta_{1}\right\}\right)=\delta A_{n+1}\left(\left\{\delta \lambda_{1}, \tilde{\lambda}_{1}, \frac{1}{\sqrt{\delta}} \eta_{1}\right\}\right) .
$$

However the analysis of this limit seems more complicated via BCFW due to the number of diagrams contributing. Instead we can consider, following [21, 45],

$$
\left\{\lambda_{1}, \tilde{\lambda}_{1}, \eta_{1}\right\} \rightarrow\left\{\sqrt{\delta} \lambda_{1}, \sqrt{\delta} \tilde{\lambda}_{1}, \sqrt{\delta} \eta_{1}\right\}
$$


Hence, after using the little scaling, we find the holomorphic limit of the superamplitude,

$$
\begin{aligned}
\lim _{\delta \rightarrow 0} A_{n+1}\left(\left\{\delta \lambda_{1}, \tilde{\lambda}_{1}, \eta_{1}\right\}\right) & =\left[\frac{1}{\delta^{2}} S^{(0)}(n, s, 2)+\frac{1}{\delta} S^{(1)}(n, s, 2)\right] A_{n} \\
& \equiv S(n, s, 2) A_{n}
\end{aligned}
$$

which defines the holomorphic soft factor $S(n, s, 2)$ given by, see [21],

$$
S^{(k)}(n, s, 2)=\frac{1}{k !} \frac{\langle n 2\rangle}{\langle n s\rangle\langle s 2\rangle}\left[\frac{\langle s n\rangle}{\langle 2 n\rangle}\left(\tilde{\lambda}_{s} \cdot \frac{\partial}{\partial \tilde{\lambda}_{2}}+\eta_{s} \cdot \frac{\partial}{\partial \eta_{2}}\right)+\frac{\langle s 2\rangle}{\langle n 2\rangle}\left(\tilde{\lambda}_{s} \cdot \frac{\partial}{\partial \tilde{\lambda}_{n}}+\eta_{s} \cdot \frac{\partial}{\partial \eta_{n}}\right)\right]^{k} .
$$

We can also consider the anti-holomorphic limit [21], under which

$$
\begin{aligned}
\lim _{\delta \rightarrow 0} A_{n+1}\left(\left\{\lambda_{1}, \delta \tilde{\lambda}_{1}, \eta_{1}\right\}\right) & =\left[\frac{1}{\delta^{2}} \bar{S}^{(0)}(n, s, 2)+\frac{1}{\delta} \bar{S}^{(1)}(n, s, 2)\right] A_{n} \\
& \equiv \bar{S}(n, s, 2) A_{n},
\end{aligned}
$$

where the anti-holomorphic soft factor is given by

$$
\bar{S}^{(k)}(n, s, 2)=\frac{1}{k !} \frac{[n 2]}{[n s][s 2]} \delta^{(4)}\left(\eta_{s}+\delta \frac{[n s]}{[2 n]} \eta_{2}+\delta \frac{[s 2]}{[2 n]} \eta_{n}\right)\left[\frac{[s n]}{[2 n]} \lambda_{s} \cdot \frac{\partial}{\partial \lambda_{2}}+\frac{[s 2]}{[n 2]} \lambda_{s} \cdot \frac{\partial}{\partial \lambda_{n}}\right]^{k} .
$$

Open Access. This article is distributed under the terms of the Creative Commons Attribution License (CC-BY 4.0), which permits any use, distribution and reproduction in any medium, provided the original author(s) and source are credited.

\section{References}

[1] F.E. Low, Bremsstrahlung of very low-energy quanta in elementary particle collisions, Phys. Rev. 110 (1958) 974 [INSPIRE].

[2] S. Weinberg, Photons and gravitons in s matrix theory: derivation of charge conservation and equality of gravitational and inertial mass, Phys. Rev. 135 (1964) B1049.

[3] T.H. Burnett and N.M. Kroll, Extension of the low soft photon theorem, Phys. Rev. Lett. 20 (1968) 86 [INSPIRE].

[4] E. Casali, Soft sub-leading divergences in Yang-Mills amplitudes, JHEP 08 (2014) 077 [arXiv: 1404.5551] [INSPIRE].

[5] F. Cachazo and A. Strominger, Evidence for a new soft graviton theorem, arXiv:1404.4091 [INSPIRE].

[6] D. Kapec, V. Lysov, S. Pasterski and A. Strominger, Semiclassical Virasoro symmetry of the quantum gravity $\mathcal{S}$-matrix, JHEP 08 (2014) 058 [arXiv: 1406.3312] [INSPIRE].

[7] A. Strominger and A. Zhiboedov, Gravitational memory, BMS supertranslations and soft theorems, arXiv:1411.5745 [INSPIRE].

[8] H. Bondi, M. van der Burg and A. Metzner, Gravitational waves in general relativity. 7. Waves from axisymmetric isolated systems, Proc. Roy. Soc. Lond. A 269 (1962) 21. 
[9] R. Sachs, Gravitational waves in general relativity. 8. Waves in asymptotically flat space-times, Proc. Roy. Soc. Lond. A 270 (1962) 103.

[10] G. Barnich and C. Troessaert, Aspects of the BMS/CFT correspondence, JHEP 05 (2010) 062 [arXiv: 1001.1541] [INSPIRE].

[11] T. He, P. Mitra and A. Strominger, 2D Kac-Moody symmetry of $4 D$ Yang-Mills theory, arXiv: 1503.02663 [INSPIRE].

[12] H. Elvang and Y.-t. Huang, Scattering amplitudes, arXiv:1308.1697 [INSPIRE].

[13] J.M. Henn and J.C. Plefka, Scattering amplitudes in gauge theories, Lecture notes in physics colume 883, Springer (2014).

[14] B.U.W. Schwab and A. Volovich, Subleading soft theorem in arbitrary dimensions from scattering equations, Phys. Rev. Lett. 113 (2014) 101601 [arXiv:1404.7749] [InSPIRE].

[15] N. Afkhami-Jeddi, Soft graviton theorem in arbitrary dimensions, arXiv:1405.3533 [INSPIRE].

[16] M. Zlotnikov, Sub-sub-leading soft-graviton theorem in arbitrary dimension, JHEP 10 (2014) 148 [arXiv: 1407.5936] [INSPIRE].

[17] C. Kalousios and F. Rojas, Next to subleading soft-graviton theorem in arbitrary dimensions, JHEP 01 (2015) 107 [arXiv:1407.5982] [INSPIRE].

[18] J. Broedel, M. de Leeuw, J. Plefka and M. Rosso, Constraining subleading soft gluon and graviton theorems, Phys. Rev. D 90 (2014) 065024 [arXiv:1406.6574] [INSPIRE].

[19] Z. Bern, S. Davies, P. Di Vecchia and J. Nohle, Low-energy behavior of gluons and gravitons from gauge invariance, Phys. Rev. D 90 (2014) 084035 [arXiv:1406.6987] [InSPIRE].

[20] Z. Bern, S. Davies and J. Nohle, On loop corrections to subleading soft behavior of gluons and gravitons, Phys. Rev. D 90 (2014) 085015 [arXiv:1405.1015] [INSPIRE].

[21] S. He, Y.-t. Huang and C. Wen, Loop corrections to soft theorems in gauge theories and gravity, JHEP 12 (2014) 115 [arXiv:1405.1410] [INSPIRE].

[22] F. Cachazo and E.Y. Yuan, Are soft theorems renormalized?, arXiv:1405.3413 [InSPIRE].

[23] M. Bianchi, S. He, Y.-t. Huang and C. Wen, More on soft theorems: trees, loops and strings, arXiv: 1406.5155 [INSPIRE].

[24] J. Broedel, M. de Leeuw, J. Plefka and M. Rosso, Local contributions to factorized soft graviton theorems at loop level, Phys. Lett. B 746 (2015) 293 [arXiv:1411.2230] [INSPIRE].

[25] Y. Geyer, A.E. Lipstein and L. Mason, Ambitwistor strings at null infinity and (subleading) soft limits, Class. Quant. Grav. 32 (2015) 055003 [arXiv:1406.1462] [INSPIRE].

[26] T. Adamo, E. Casali and D. Skinner, Perturbative gravity at null infinity, Class. Quant. Grav. 31 (2014) 225008 [arXiv:1405.5122] [INSPIRE].

[27] F. Cachazo, S. He and E.Y. Yuan, Scattering of massless particles in arbitrary dimensions, Phys. Rev. Lett. 113 (2014) 171601 [arXiv:1307.2199] [INSPIRE].

[28] F. Cachazo, S. He and E.Y. Yuan, Scattering of massless particles: scalars, gluons and gravitons, JHEP 07 (2014) 033 [arXiv:1309.0885] [INSPIRE].

[29] A.E. Lipstein, Soft theorems from conformal field theory, JHEP 06 (2015) 166 [arXiv: 1504.01364] [INSPIRE]. 
[30] S.L. Adler, Consistency conditions on the strong interactions implied by a partially conserved axial vector current, Phys. Rev. 137 (1965) B1022.

[31] N. Arkani-Hamed, F. Cachazo and J. Kaplan, What is the simplest quantum field theory?, JHEP 09 (2010) 016 [arXiv:0808.1446] [INSPIRE].

[32] W.-M. Chen, Y.-t. Huang and C. Wen, From U(1) to E8 : soft theorems in supergravity amplitudes, JHEP 03 (2015) 150 [arXiv:1412.1811] [INSPIRE].

[33] C. Cheung, K. Kampf, J. Novotny and J. Trnka, Effective field theories from soft limits of scattering amplitudes, Phys. Rev. Lett. 114 (2015) 221602 [arXiv:1412.4095] [INSPIRE].

[34] W.-M. Chen, Y.-t. Huang and C. Wen, New fermionic soft theorems, Phys. Rev. Lett. 115 (2015) 021603 [arXiv: 1412.1809] [INSPIRE].

[35] F. Cachazo, S. He and E.Y. Yuan, New double soft emission theorems, arXiv:1503.04816 [INSPIRE].

[36] A. Brandhuber, P. Heslop and G. Travaglini, A note on dual superconformal symmetry of the $N=4$ super Yang-Mills S-matrix, Phys. Rev. D 78 (2008) 125005 [arXiv:0807.4097] [INSPIRE].

[37] A. Volovich, C. Wen and M. Zlotnikov, Double soft theorems in gauge and string theories, arXiv: 1504.05559 [INSPIRE].

[38] D. Maître and P. Mastrolia, S@M, a Mathematica implementation of the spinor-helicity formalism, Comput. Phys. Commun. 179 (2008) 501 [arXiv:0710.5559] [INSPIRE].

[39] L.J. Dixon, J.M. Henn, J. Plefka and T. Schuster, All tree-level amplitudes in massless QCD, JHEP 01 (2011) 035 [arXiv: 1010.3991] [INSPIRE].

[40] T. Schuster, Color ordering in QCD, Phys. Rev. D 89 (2014) 105022 [arXiv:1311.6296] [INSPIRE].

[41] R. Britto, F. Cachazo and B. Feng, New recursion relations for tree amplitudes of gluons, Nucl. Phys. B 715 (2005) 499 [hep-th/0412308] [INSPIRE].

[42] R. Britto, F. Cachazo, B. Feng and E. Witten, Direct proof of tree-level recursion relation in Yang-Mills theory, Phys. Rev. Lett. 94 (2005) 181602 [hep-th/0501052] [INSPIRE].

[43] J. Bedford, A. Brandhuber, B.J. Spence and G. Travaglini, A recursion relation for gravity amplitudes, Nucl. Phys. B 721 (2005) 98 [hep-th/0502146] [INSPIRE].

[44] F. Cachazo and P. Svrček, Tree level recursion relations in general relativity, hep-th/0502160 [INSPIRE].

[45] D. Nandan and C. Wen, Generating all tree amplitudes in $N=4 S Y M$ by inverse soft limit, JHEP 08 (2012) 040 [arXiv: 1204.4841] [INSPIRE]. 